\title{
Structure
}

\section{Structure-based modeling and dynamics of MurM, a Streptococcus pneumoniae penicillin resistance determinant present at the cytoplasmic membrane}

\section{Graphical abstract}

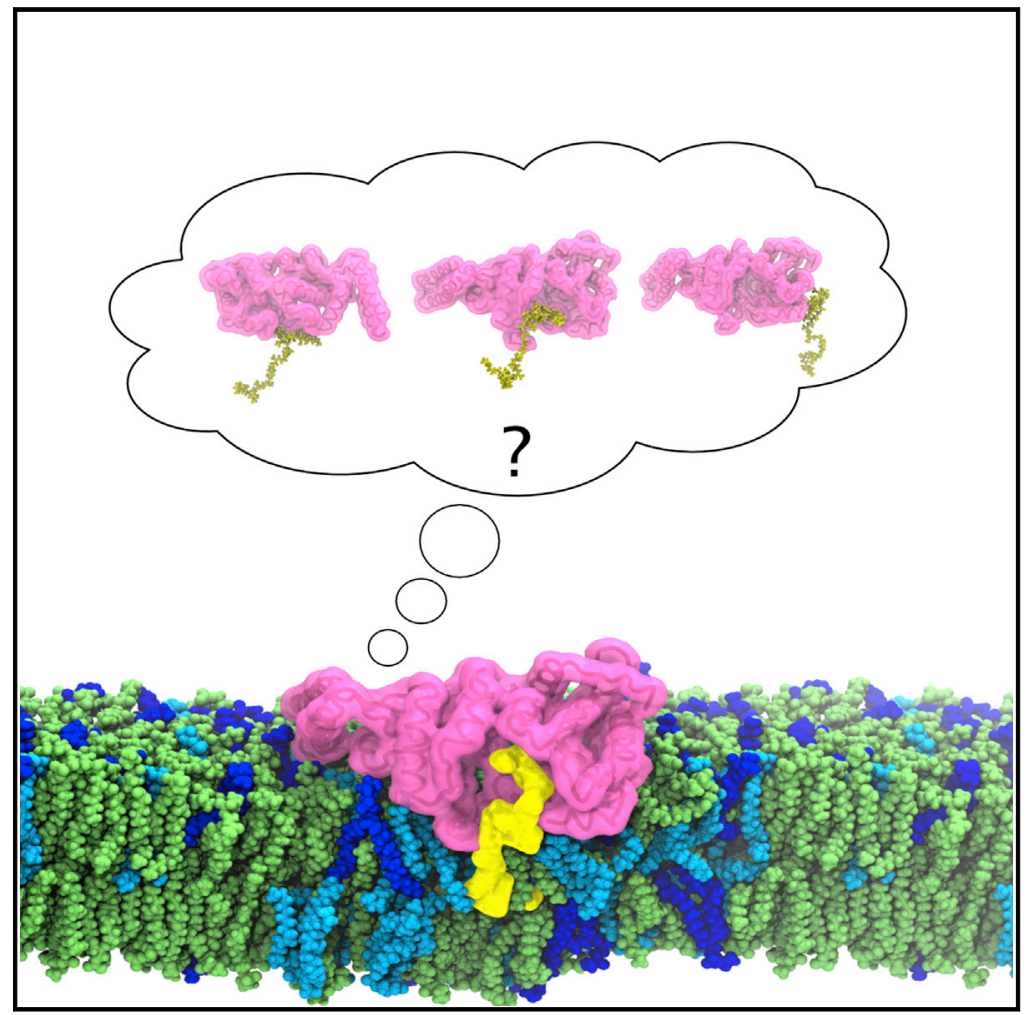

\section{Highlights}

- S. aureus FemX structure generated an improved homology model of S. pneumoniae MurM

- A Lipid II binding site in MurM was identified by docking and molecular dynamics

- Modeling indicates cardiolipin is enriched at the MurM protein:membrane interface

- Enzyme assays show that phospholipids influence MurM activity

\section{Authors}

Anna York, Adrian.J. Lloyd,

Charo I. del Genio, ....,

Christopher.G. Dowson, Syma Khalid, David.I. Roper

\section{Correspondence}

s.khalid@soton.ac.uk (S.K.), david.roper@warwick.ac.uk (D.I.R.)

\section{In brief}

With an improved homology model of MurM, York et al., use docking and molecular dynamics studies to identify a potential Lipid II binding site and simulate how MurM interacts with this membraneembedded substrate. Cardiolipin, a membrane phospholipid, was also found to associate with MurM and upregulate its enzymatic activity. 


\title{
Structure-based modeling and dynamics of MurM, a Streptococcus pneumoniae penicillin resistance determinant present at the cytoplasmic membrane
}

\author{
Anna York, ${ }^{1,5}$ Adrian.J. Lloyd, ${ }^{1,5}$ Charo I. del Genio, ${ }^{2}$ Jonathan Shearer, ${ }^{3}$ Karen.J. Hinxman, ${ }^{1}$ Konstantin Fritz, ${ }^{1}$ \\ Vilmos Fulop, ${ }^{1}$ Christopher.G. Dowson, ${ }^{1}$ Syma Khalid, ${ }^{3, *}$ and David.I. Roper ${ }^{1,4, *}$ \\ ${ }^{1}$ School of Life Science, University of Warwick, Coventry, West Midlands CV4 7AL, UK \\ ${ }^{2}$ Centre for Fluid and Complex Systems, School of Computing, Electronics and Mathematics, University of Coventry, West Midlands CV1 \\ $5 \mathrm{FB}, \mathrm{UK}$ \\ ${ }^{3}$ School of Chemistry, University of Southampton, Southampton, Hampshire SO17 1BJ, UK \\ ${ }^{4}$ Department of Physiology and Cellular Biophysics, Columbia University Irving Medical Center, New York, NY, USA \\ 5These authors contributed equally \\ ${ }^{*}$ Correspondence: s.khalid@soton.ac.uk (S.K.), david.roper@warwick.ac.uk (D.I.R.) \\ https://doi.org/10.1016/j.str.2021.03.001
}

\section{SUMMARY}

Branched Lipid II, required for the formation of indirectly crosslinked peptidoglycan, is generated by MurM, a protein essential for high-level penicillin resistance in the human pathogen Streptococcus pneumoniae. We have solved the X-ray crystal structure of Staphylococcus aureus FemX, an isofunctional homolog, and have used this as a template to generate a MurM homology model. Using this model, we perform molecular docking and molecular dynamics to examine the interaction of MurM with the phospholipid bilayer and the membrane-embedded Lipid II substrate. Our model suggests that MurM is associated with the major membrane phospholipid cardiolipin, and experimental evidence confirms that the activity of MurM is enhanced by this phospholipid and inhibited by its direct precursor phosphatidylglycerol. The spatial association of pneumococcal membrane phospholipids and their impact on MurM activity may therefore be critical to the final architecture of peptidoglycan and the expression of clinically relevant penicillin resistance in this pathogen.

\section{INTRODUCTION}

The peptidoglycan (PG) of the bacterial cell wall is a polymer consisting of alternating $\beta$-1,4-linked $N$-acetyl glucosamine (GlcNAc) and $N$-acetyl muramic acid (MurNAc) residues. Appended to the MurNAc sugar is a pentapeptide stem that can be crosslinked directly or indirectly to form a rigid mesh-like structure (Bugg et al., 2011). PG biosynthesis begins with the cytoplasmic formation of a Park nucleotide, which is subsequently converted into a lipid-linked PG precursor known as Lipid II. Lipid II is then transported across the membrane, where it is polymerized and crosslinked by the penicillin-binding proteins (PBPs) (Figure 1). PG is an essential component of the cell wall, involved in cell growth and division, maintaining structural integrity, and resisting high osmotic pressures. Inhibition of cell wall biosynthesis is a key mechanism for many antibiotics, including $\beta$-lactams, glycopeptides, and amino acid analogs (Schneider and Sahl, 2010).

In Streptococcus pneumoniae and other Gram-positive bacteria, the glutamate at the second position of the Lipid II pentapeptide is $\alpha$-amidated to form iso-glutamine by the essential GatT/ MurD complex (Figueiredo et al., 2012; Münch et al., 2012; Zapun et al., 2013; Morlot et al., 2018). In addition, branched Lipid
II, capable of generating indirect crosslinks, can be formed by the non-essential aminoacyl-tRNA-dependent ligases, MurM and MurN. MurM and MurN are responsible for the sequential addition of amino acids to the third-position lysine of the pentapeptide stem of Lipid II (Filipe et al., 2000). MurM can append either L-serine or L-alanine at the first position of the dipeptide bridge, while MurN extends this modification by addition of an invariable L-alanyl moiety. Branched PG precursors are also found in several other Gram-positive bacterial pathogens, for example the glycyl-tRNA ${ }^{\text {Gly }}$-dependent enzymes FemX, FemA, and FemB are responsible for the addition of a pentaglycyl bridge in Staphylococcus aureus (Schneider et al., 2004). In comparison with other Gram-positive organisms, the PG of S. pneumoniae is highly heterogeneous: the predominant C-terminal amino acid at position 1 of the dipeptide and the proportion of indirect crosslinks throughout the PG vary significantly between different strains (Severin and Tomasz, 1996; Garcia-Bustos et al., 1987; Garcia-Bustos and Tomasz, 1990). In vitro and in vivo studies indicate that MurM from the penicillin-resistant strain $S$. pneumoniae(159) preferentially incorporates L-alanine, while MurM from a penicillin-sensitive strain $S$. pneumoniae(Pn16) preferentially incorporates L-serine (Lloyd et al., 2008). In addition, penicillin-resistant strains demonstrated higher levels of 


\section{Peptidoglycan Biosynthesis Pathway in Streptococcus pneumoniae}

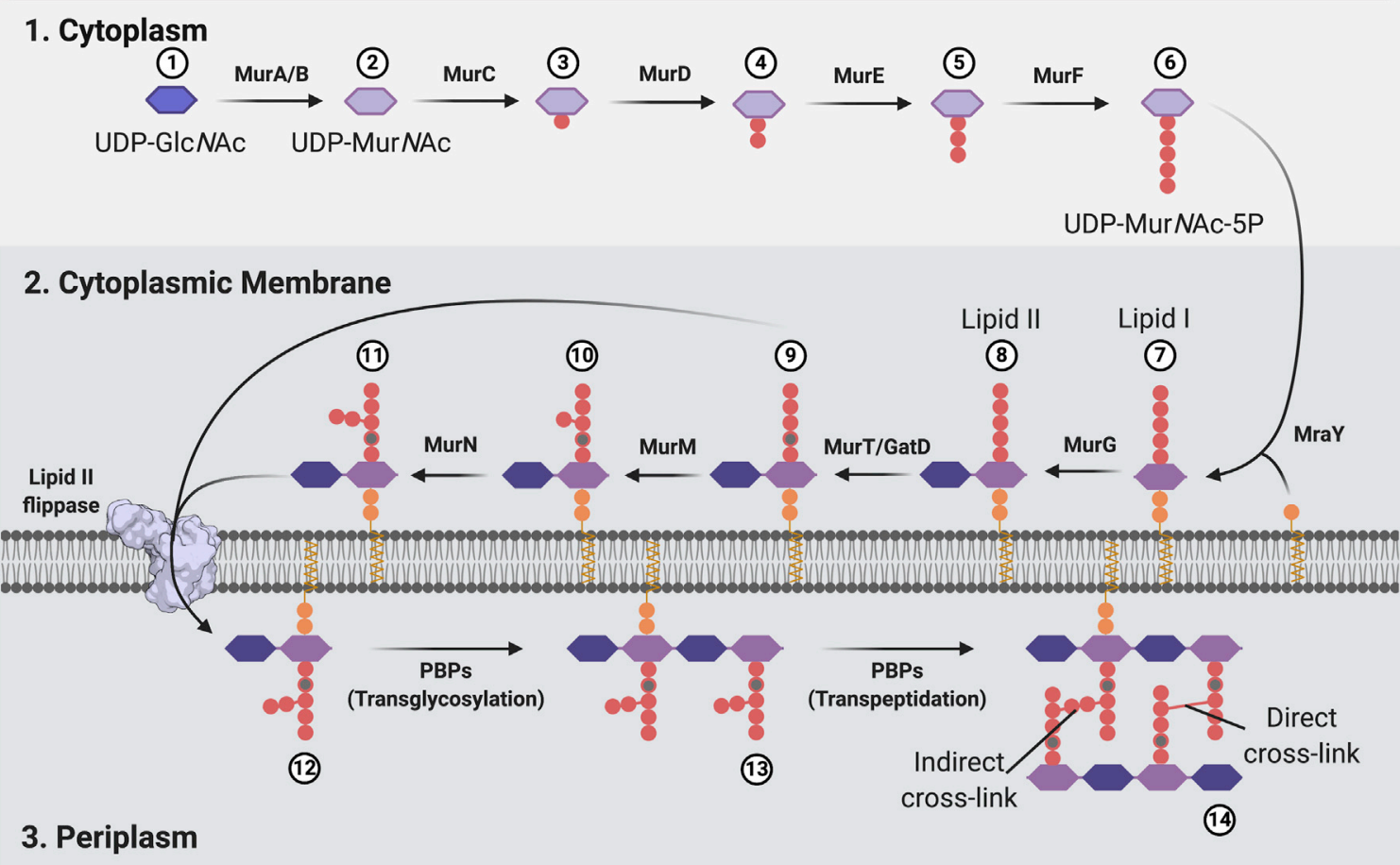

Figure 1. The PG biosynthesis pathway

(1) The cytoplasmic stage is characterized by the formation of UDP-MurNAc-pentapeptide (UDP-MurNAc-5P) by MurA-MurF. The pentapeptide stem peptide usually comprises L-Ala- $\gamma$-D-Glu-L-Lys-D-Ala-D-Ala in most Gram-positive organisms including the pneumococcus.

(2) At the internal face of the cytoplasmic membrane, MraY catalyzes the formation of Lipid I from UDP-MurNAc-5P to undecaprenyl-pyrophosphate, which is then converted to Lipid II by MurG. In S. pneumoniae, the second position D-glutamyl $\alpha$-carboxyl is amidated to D-iso-glutamine (iGln) by the MurT/GatD complex, and in some cases a dipeptide branch of either L-Ser/L-Ala or L-Ala/L-Ala may be appended at the $\varepsilon$-amino group of the third-position lysine by MurM and MurN, respectively. The exact order of the cytoplasmic membrane steps remains uncertain, but here for clarity they appear as a linear sequence, with conversion to Lipid II occurring before peptide stem modifications, and amidation occurring before branching. Lipid II is translocated across the membrane by MurJ.

(3) At the external face of the cytoplasmic membrane, class A PBPs and complexes of SEDs-Class B PBPs form glycan chains by transglycosylation (TG), with the concomitant release of undecaprenyl-pyrophosphate, and form either direct or indirect crosslinks throughout the PG layer via transpeptidation (TP). Nucleotide sugars UDP-GICNAc and UDP-MurNAc and the sugars GIcNAc and MurNAc are signified by blue, violet, dark-blue, and purple elongated hexagons, respectively. Figure created using BioRender.com.

indirect crosslinking in the PG compared with penicillin-susceptible isolates, although the overall degree of crosslinking remained constant (Garcia-Bustos and Tomasz, 1990).

Resistance to $\beta$-lactam antibiotics in S. pneumoniae is characterized by extensive interspecies recombination of PBP transpeptidase domains, which results in sequence heterogeneity within and around the mosaic PBP active site with a consequential lowering of $\beta$-lactam binding affinity (Smith et al., 1991). This mechanism of resistance contrasts with that of many other bacteria that have acquired genes for $\beta$-lactamase enzymes which inactivate the antibiotic before it binds to and inhibits the PBPs. Interestingly, deletion of the murM gene in $S$. pneumoniae eliminates indirect crosslinks from the $P G$ and results in a complete loss of penicillin resistance (Filipe et al., 2001). It has been proposed that the changes to the PBP active site, which prevent $\beta$-lactam binding, may also alter the Lipid II substrate specificity such that the PBPs bind branched Lipid II more tightly than unbranched
Lipid II. MurM is therefore necessary but not sufficient for resistance in clinical strains of $S$. pneumoniae, making it an interesting target for the development of new inhibitors of antimicrobial resistance (Filipe and Tomasz, 2000).

The cytoplasmic membrane of $S$. pneumoniae contains two phospholipids, phosphatidylglycerol and cardiolipin (Trombe et al., 1979; Pesakhov et al., 2007), where cardiolipin synthase is responsible for generating cardiolipin from two molecules of phosphatidylglycerol (Schlame, 2008). The proportion of cardiolipin and phosphatidylglycerol, as a percentage of the overall membrane lipids, varies in $S$. pneumoniae between anaerobic and aerobic growth conditions. Cardiolipin was found to decrease from $15.3 \%$ to $8.3 \%$ while phosphatidylglycerol increased from $12.7 \%$ to $16.3 \%$ in anaerobic conditions compared with aerobic conditions (Pesakhov et al., 2007). The peptidoglycan precursor, Lipid II, is tethered to the cell membrane by virtue of its C55 Lipid II tail. Therefore, MurM acts on its lipid substrate in close proximity to the cytoplasmic leaflet 
Table 1. Summary of crystallographic data collection and refinement statistics from the $S$. aureus FemX structure

\begin{tabular}{ll}
\hline & FemX \\
\hline Data collection & \\
\hline $\begin{array}{l}\text { Synchrotron radiation detector, } \\
\text { wavelength }(\AA)\end{array}$ & Pilatus $6 \mathrm{M}-\mathrm{F}, 0.920$ \\
Unit cell $a, b, c(\AA), \alpha, \beta, \gamma\left(^{\circ}\right)$ & $45.01,83.62,133.93,90.0$, \\
& $90.0,90.0$ \\
Space group & $\mathrm{P} 2{ }_{1} 2{ }_{1}{ }_{1}$ \\
Resolution $(\AA)$ & $52.27-1.62[1.66-1.62]$ \\
Observations & $422,822[29,596]$ \\
Unique reflections & $65,058[4,782]$ \\
$l / \sigma(I)$ & $15.7[2.6]$ \\
$R_{\text {sym }}{ }^{a}$ & $0.065[0.567]$ \\
$R_{\text {meas }}$ & $0.078[0.690]$ \\
$R_{\text {p.i.m }}$ & $0.031[0.273]$ \\
Completeness (\%) & $99.7[99.8]$
\end{tabular}

\begin{tabular}{|c|c|}
\hline Refinement & \\
\hline Non-hydrogen atoms & 3,397 (including 177 waters) \\
\hline$R_{\text {cryst }}^{\mathrm{b}}$ & $0.221[0.262]$ \\
\hline Reflections used & $61,691[4,531]$ \\
\hline$R_{\text {free }}{ }^{\mathrm{c}}$ & $0.262[0.296]$ \\
\hline Reflections used & $3,294[244]$ \\
\hline$R_{\text {cryst }}$ (all data) $)^{\mathrm{b}}$ & 0.222 \\
\hline Average temperature factor $\left(\AA^{2}\right)$ & 26 \\
\hline \multicolumn{2}{|l|}{ RMSDs from ideal values } \\
\hline Bonds $(\AA ̊)$ & 0.013 \\
\hline Angles $\left(^{\circ}\right)$ & 1.5 \\
\hline DPI coordinate error $(\AA)^{d}$ & 0.098 \\
\hline \multicolumn{2}{|l|}{ Ramachandran plot ${ }^{\mathrm{e}}$} \\
\hline Favored (\%) & 98.0 \\
\hline Outliers (\%) & 0.0 \\
\hline
\end{tabular}

The highest-resolution bin of data is indicated by square brackets. Numbers in square brackets refer to values in the highest-resolution shell.

${ }^{\mathrm{a}} R_{\text {sym }}=S_{j} S_{h}\left|I_{h, j}-\left\langle I_{h}\right\rangle / S_{j} S_{h}<I_{h}\right\rangle$, where $I_{h, j}$ is the is the $j$ th observation of reflection $h$, and $I_{h}$ is the mean intensity of that reflection.

${ }^{\mathrm{b}} R_{\text {cryst }}=S|| F_{\text {obs }}|-| F_{\text {calc }} \| / S\left|F_{\text {obs }}\right|$, where $F_{\text {obs }}$ and $F_{\text {calc }}$ are the observed and calculated structure factor amplitudes, respectively.

${ }^{\mathrm{c}} R_{\text {free }}$ is equivalent to $R_{\text {cryst }}$ for a $4 \%$ subset of reflections not used in the refinement (Brünger, 1992).

${ }^{\mathrm{d} D P I}$ refers to the diffraction component precision index (Cruickshank, 1999).

${ }^{\mathrm{e}}$ As calculated by MolProbity (Williams et al., 2008).

of the cell membrane and is potentially influenced by membrane phospholipid composition.

Previously, MurM inhibitors have been identified; however, none have shown growth inhibition or any effect on penicillin minimum inhibitory concentration, indicating that these compounds cannot effectively cross the cytoplasmic membrane of S. pneumoniae (Cressina et al., 2007, 2009). MurM has thus far resisted extensive crystallization in our laboratory, and consequently its X-ray-solved structure is not available. However, in a related study we were able to solve the X-ray structure of the isofunctional homolog of MurM from S. aureus (FemX), which we have used here as a template for homology modeling of MurM. Using this MurM homology model, we have successfully identified the Lipid II binding site and have used molecular dynamics (MD) simulations to investigate interactions between MurM and both membrane phospholipids and its Lipid II substrate (Witzke et al., 2016). We subsequently studied the effects of these membrane-embedded phospholipids, on the enzymatic activity of MurM in vitro, corroborating our in silico analysis. These studies provide insights into the structure and activity of MurM, providing a link between phospholipid membrane composition and peptidoglycan architecture. This may be useful for the development of chemical probes for these proteins and has important implications for future studies on penicillin resistance mechanisms in S. pneumoniae.

\section{RESULTS}

X-ray crystallography and structure determination of S. aureus FemX

The crystal structure of $S$. aureus FemX was solved to a resolution of $1.62 \AA$, and the structure was deposited in the PDB with accession number PDB: 6SNR. A summary of the data collection and refinement statistics is given in Table 1.

The final solved structure of $S$. aureus FemX contains a globular domain and a coiled-coil domain. Similar to FemA (Benson et al., 2002), the globular domain can be divided into two subdomains. Each subdomain contains a central five-stranded mixedpolarity $\beta$ sheet surrounded by four $\alpha$ helices. Subdomain $1 \mathrm{~A}$ comprises residues $1-145$ and $384-421$ while subdomain $1 \mathrm{~B}$ comprises residues 146-234 and 298-383. Unfortunately, residues 403-421 were not present in the density. The coiled-coil domain consists of two antiparallel $\alpha$ helices, comprising residues 235-297. FemX and FemA can be superimposed onto each other with a root-mean-square deviation (RMSD) of $\sim 2.7 \AA$ over 384 residues. Similarly to FemA, FemX has a deep L-shaped channel of about $20 \times 40 \AA$ located alongside the globular domain and mainly in subdomain $1 \mathrm{~B}$. This channel comprises a peptidoglycan precursor binding site which was previously identified in S. aureus FemA (Benson et al., 2002). The identity of this peptidoglycan precursor binding site has been further confirmed by crystallographic analysis of Weissella viridescens FemX complexed with substrates (Biarrotte-Sorin et al., 2004).

\section{Homology modeling of S. pneumoniae MurM}

The structures of two MurM homologs, S. aureus FemA and $W$. viridescens FemX, were solved previously by X-ray crystallography (Benson et al., 2002; Fonvielle et al., 2013; Biarrotte-Sorin et al., 2004). The $S$. aureus FemA structure was subsequently used as a template for homology modeling of MurM by Fiser et al. (2003). However, alignment of S. aureus FemA, S. aureus FemX, S. pneumoniae MurM, and W. viridescens FemX (Figure $S 1$ ) showed that $S$. aureus FemX possesses the highest sequence identity to MurM and is also more functionally homologous to S. pneumoniae MurM, as it appends the first amino acid of the cross-bridge to the Lipid II precursor (Matsuhashi et al., 1967; Schneider et al., 2004). In contrast, S. aureus FemA appends the second and third amino acid residues of the crossbridge to the $\alpha$-amino group of a glycyl residue appended to 


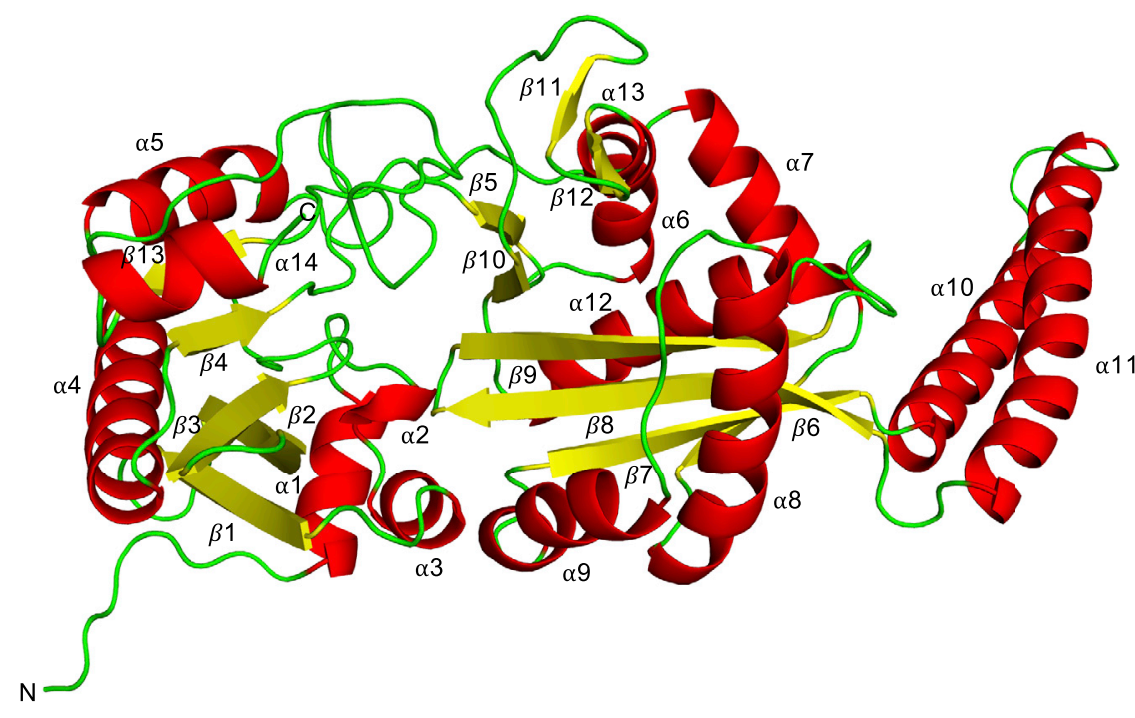

Figure 2. Cartoon representation of MurM predicted structure

Fourteen $\alpha$ helices (red), $12 \beta$ sheets (yellow), and unstructured regions (green). Best model obtained based on SOAP and DOPE scores following homology modeling using MODELLER with $S$. aureus FemX as a template. The structure was rendered in PyMOL (version 2.2.0).

sponding MurM residues, defined as having residues which have similar properties, and occupying a similar location and orientation in physical space with side chains facing the binding pocket, were identified in the MurM structure as Phe103, Lys35, Trp38, Arg215, and Tyr219; therefore, these residues may also be important for substrate binding in MurM.

the $\varepsilon$-amino group of the stem peptide L-lysyl residue of the Lipid II precursor. Therefore, given the difficulties in obtaining MurM crystals, we were motivated to solve the structure of its functional homolog (S. aureus FemX) by X-ray crystallography to access the structure of MurM in silico.

Using the structure of $S$. aureus FemX, we generated a homology model for MurM, which consists of a globular domain comprising two subdomains and a coiled-coil helical arm (Figure 2). Each subdomain comprises two twisted $\beta$-sheet cores surrounded by $\alpha$ helices; subdomain $1 \mathrm{~A}$ is formed of residues 1-153 and 382-401, while subdomain $1 \mathrm{~B}$ is made up of residues 154-241 and 294-381. The coiled-coil domain comprises residues 242-293. While the MurM homology model presented here is similar to the previous model (Fiser et al., 2003), the RMSD of the two models is $3.8 \AA$ over 368 residues, indicating that there are some key structural differences, namely: loss of $\mathrm{N}$-terminal $\beta 1$ and antiparallel $\beta 6 / \beta 13$ from the previous model; addition of $\alpha 5$ and $\beta 11 / \beta 12$; and presence of an $\alpha$-helical secondary structure proximal to the C-terminal end of this MurM model.

Identification of a possible Lipid II binding site of MurM Our MurM model revealed a binding pocket that was not present in the previous model of MurM produced by Fiser et al. (2003). Structural comparison between $W$. viridescens FemX co-crystallized with its uridine diphosphate (UDP)-MurNAc-pentapeptide substrate and this MurM model allowed identification of a Lipid II binding site that corresponds to the peptidoglycan precursor binding sites identified previously in $S$. aureus FemA and W. viridescens FemX (Benson et al., 2002; Biarrotte-Sorin et al., 2004) and in our structure of $S$. aureus FemX. When this MurM model and the $W$. viridescens FemX were aligned and overlaid, the proposed MurM binding site appeared to easily accommodate the soluble UDP-MurNAc-pentapeptide substrate well (Figure 3A). The following eight residues-Tyr103, Lys36, Asn38, Trp39, Thr209, Arg211, Try215 and Tyr256-were independently proposed to be involved in substrate binding in the peptidoglycan precursor and tRNA liganded $W$. viridescens FemX structures (Biarrotte-Sorin et al., 2004; Fonvielle et al., 2013). The corre-
Next, molecular docking using AutoDock Vina (Trott and Olson, 2010) was conducted to independently investigate docking of the Lipid II substrate to the MurM model we generated. Lipid II is a large molecule that is, in general, unsuitable for molecular membrane, and so is not itself available for binding to MurM. Therefore, a truncated Lipid II molecule, composed of a methyl capped diphospho GlcNAc-MurNAc-pentapeptide, was used for these docking experiments (Figure S2).

When AutoDock Vina was allowed to search the entire protein surface of MurM, all docking results returned were within the identified binding site, indicating that there are no other suitable binding sites on the protein. The search was then restricted to the binding site, and the top ten results were obtained. The top five results obtained all had identical binding affinities; however, two of these docking orientations, where the phosphates are located deep within the binding pocket, would be physically impossible for the natural substrate (Lipid II) in vivo, since the membrane-embedded prenyl lipid tail is appended via the phosphate. The remaining three docking orientations all orient the phosphates close to the opening of the binding site with the pentapeptide chain disappearing deep into the binding pocket. The exact orientation of the pentapeptide chain is variable, indicating that the binding site is spacious and that Lipid II may be accommodated in a number of different possible orientations. Figure $3 \mathrm{~B}$ shows one conformation in which the docking of truncated Lipid II is similar to the orientation of the soluble UDP-MurNAc-pentapeptide from $W$. viridescens FemX overlaid with MurM (Figure 3A), the remaining four substrate orientations are shown in Figure S3. A key limitation of docking is that it considers the protein as rigid; therefore, multiple substrate orientations may indicate that conformational changes within the binding site may occur upon substrate binding or during catalysis.

Additional docking studies were performed to further explore the Lipid II binding pose in the putative MurM binding site and establish whether this binding site shows specific affinity for Lipid II or is indiscriminate between different lipid species. Therefore, Lipid II, cardiolipin, phosphatidylglycerol, and phosphatidylethanolamine lipids (full molecules) were docked into the docking studies. In addition, the lipid tail is embedded in the 


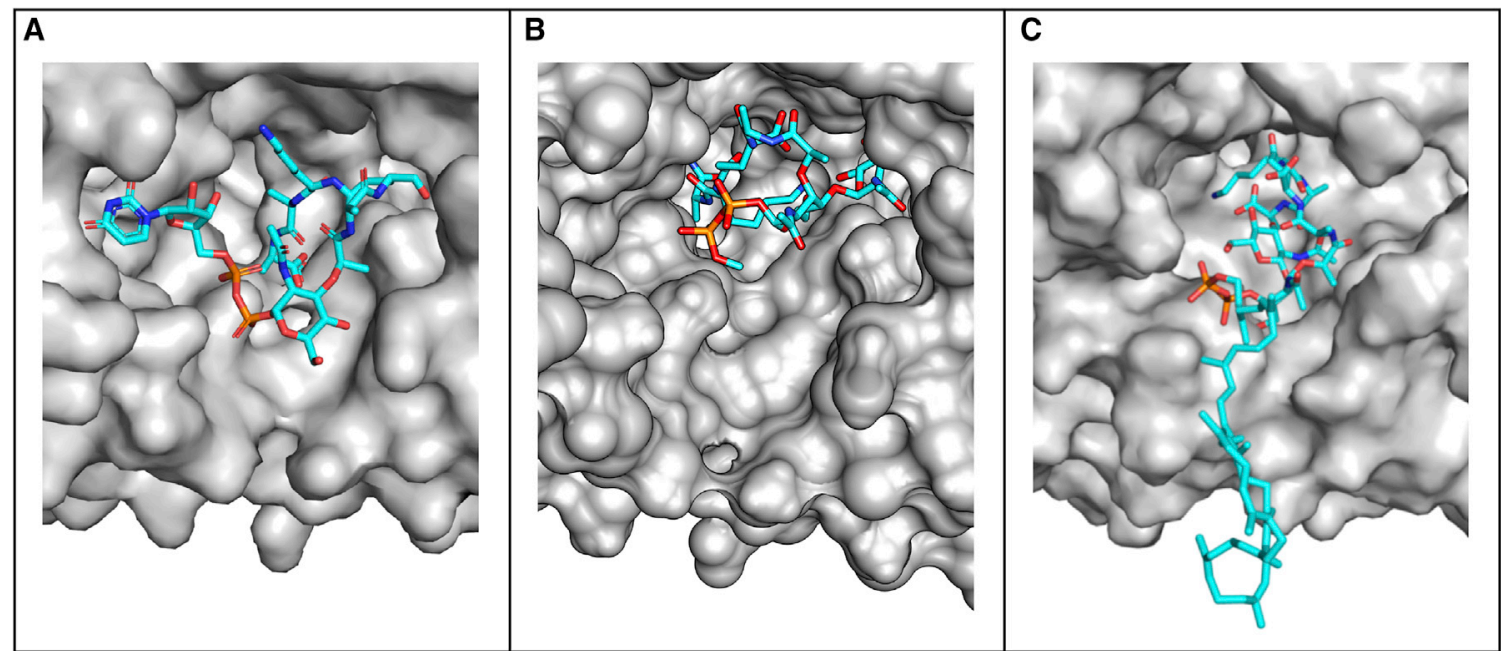

Figure 3. Surface representation of MurM binding site

(A) MurM $_{159}$ model aligned and overlaid with the UDP-MurNAc-pentapeptide substrate, which was co-crystallized with W. viridescens FemX.

(B) MurM ${ }_{159}$ model with truncated Lipid II docked in the binding site, using AutoDock Vina.

(C) MurM ${ }_{159}$ model with Lipid II in the binding site, from membrane simulations. Figures were created with PyMOL (version 2.2.0) and Chimera (version 1.13.1). See also Figures S2 and S3.

putative binding site, where the binding site is defined by the residues Lys35, Trp38, Phe103, Arg215, and Tyr219. We found Lipid II bound into this site with atoms of the headgroup within $3 \AA$ of the residues Lys35, Trp38, Phe215, and Arg219, and with the Lipid II tail outside of this site. In stark contrast, the cardiolipin and phosphatidylethanolamine headgroups did not show any preference for this site; instead the binding poses showed their tails inserted into the cavity. There was one binding pose for phosphatidylglycerol whereby the phospholipid headgroup was in the putative binding site, although a portion of the tail was also inside it; the remaining two phosphatidylglycerol poses had the tails inserted into this cavity. For each lipid type the top cluster of binding poses produced by HADDOCK2.4 (Van Zundert et al., 2016) were analyzed, and the top three binding poses for each lipid are shown in Figure S4.

\section{Interactions between MurM and the lipid bilayer}

Given the docking studies are performed in the absence of the membrane environment, a set of coarse-grained MD simulations was next performed to establish the likelihood of MurM being oriented on the membrane such that the putative binding site is available to Lipid II. MD simulations were used to model the interactions of MurM with the lipid bilayer. Six independent coarsegrained simulations were conducted for each of three membrane systems described in Table S1. In 16 of these simulation runs, MurM readily associated with the membrane in $<3 \mu$ s and its orientation with respect to the membrane remained unchanged for the remaining $2 \mu \mathrm{s}$ (Figure S5). In addition, in the same membrane leaflet as the peripheral MurM, more than $50 \%$ of Lipid II molecules were located within $2 \mathrm{~nm}$ of the protein. MurM associated with the membrane in a number of different orientations, which can be classified into two groups: those in which the binding site predicted from docking studies is available for lipid binding and those in which it is not. Table 2 shows that in 18 unbiased simulations; MurM adhered to the membrane in 16 of the simulations and of these, the binding site was available for Lipid II binding in 11 and unavailable in only 5 . In 3 of these 18 unbiased simulations, MurM was oriented such that the Lipid II molecule was located in the putative binding site, which demonstrates that Lipid II is able to successfully enter this binding site even on the short timescale of an MD simulation (Figure 4). Back mapping of one of these systems to all-atom resolution allowed the binding site to be explored in more detail. Three independent atomistic simulations each of 250 ns duration found Lipid II located in the same binding site of MurM that was identified during molecular docking of the truncated Lipid II substrate, and in the co-crystal structure of UDP-MurNAc-pentapeptide with $W$. viridescens FemX (Figure $3 \mathrm{~A}$ ). In both the molecular docking and atomistic simulations, the Lipid II headgroup forms stable interactions with Lys35, Trp38, Arg215, and Tyr219.

Similarly to the molecular docking findings, this MD simulation shows that the MurM binding site is flexible and allows the Lipid II molecule to adopt a wide variety of conformations (Figure 5). This may suggest that binding of a second substrate or a large conformational change may be required for catalysis.

\section{Interactions between MurM and membrane} phospholipids

MD simulations were used to investigate the effects of membrane phospholipids (cardiolipin and phosphatidylglycerol) on MurM at the cytoplasmic membrane interface. To investigate local lipid enrichment/depletion, we calculated two-dimensional enrichment maps across the entire membrane and depletionenrichment (D-E) indices within $1.1 \mathrm{~nm}$ of MurM, as described by Corradi et al. (2018) (see STAR Methods). A D-E index $<0$, or an enrichment percentage $<0 \%$, indicated that the specified lipid was depleted with respect to the bulk membrane composition. Figure 6 shows that upon association of MurM with the 
Table 2. Summary of MurM adherence to and orientation on the three different membrane systems

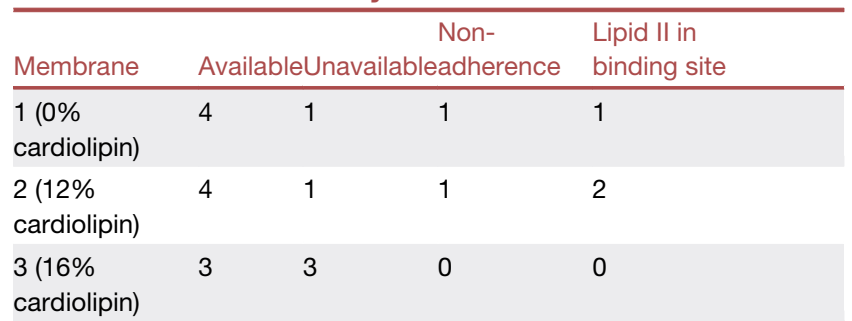

Orientation of MurM was categorized such that the putative binding site was either available for Lipid II binding (facing/close to the membrane) or unavailable for Lipid II binding (facing away from the membrane).

cytoplasmic membrane, there was no effect on the distribution of phosphatidylglycerol or phosphatidylethanolamine. However, in membranes containing $8 \%$ or $16 \%$ cardiolipin, cardiolipin was enriched at the MurM:membrane interface.

The importance of these observations was considered in vitro by measuring the enzymatic activity of MurM in the presence of varying concentrations of cardiolipin or phosphatidylglycerol. These enzymatic studies show that cardiolipin activates MurM while phosphatidylglycerol inhibits MurM in a concentrationdependent manner. Figure $6 \mathrm{E}$ shows the enzymatic activation of MurM with respect to cardiolipin concentration whereby a 9.1-fold activation of MurM was achieved, with $50 \%$ activation occurring at $0.4 \mathrm{mM}$ cardiolipin. Figure $6 \mathrm{~F}$ shows that the activity of MurM could be completely inhibited by phosphatidylglycerol, with an $\mathrm{IC}_{50}$ of $0.2 \mathrm{mM}$. Furthermore, Hill coefficients of $2.7 \pm 0.3$ and $2.8 \pm 0.2$ for cardiolipin and phosphatidylglycerol, respectively, indicate that both these phospholipids exhibit their effects on MurM in a cooperative manner. Phosphatidylethanolamine, used in the construction of the model pneumococcal membrane to which MurM bound, when tested at a concentration of $0.72 \mathrm{mM}$, only slightly activated MurM activity by 0.32 -fold (duplicate determination with a difference of $<10 \%$ ). In comparison, $0.72 \mathrm{mM}$ cardiolipin activated MurM by 8 -fold (Figure 6E). Therefore, the impact of phosphatidylethanolamine on the disposition of MurM relative to its interaction with Lipid II and the phospholipid bilayer could be neglected.

\section{DISCUSSION}

The crystal structure of $S$. aureus FemX has allowed us to generate an improved homology model of MurM leading to the identification of a putative Lipid II binding site. Fiser et al. (2003) proposed a different MurM model and speculated about an alternative binding site based on structural and functional analogy between MurM and $N$-myristoyltransferase (NMT) proteins. However, while the substrates of both NMT proteins and MurM are lipids, they are contextually very different. The NMT proteins are cytoplasmic proteins that contain a deep, narrow pocket which is highly specific for the myristoyl fatty acyl chain (Wright et al., 2010; Heuckeroth et al., 1988). In contrast, MurM binds the disaccharide headgroup and pentapeptide side chain of Lipid II, and the undecaprenyl C55 lipid tail is embedded in the membrane. Despite similarities with NMT proteins, the substrate binding site we propose more closely resembles those of $W$. viridescens FemX, S. aureus FemX, and FemA.

The orientations of truncated Lipid II in docking studies and Lipid II in MD simulations are strikingly similar to each other and also the orientation of UDP-MurNAc-pentapeptide substrate co-crystallized in $W$. viridescens FemX. This is consistent with the observation that, although inefficient compared with Lipid II, UDP-MurNAc pentapeptide is a MurM substrate (Lloyd et al., 2008). In all cases, the diphosphates are near the surface of the protein, and the protruding pentapeptide reaches into the binding pocket with the third-position lysine on the left-hand side of the binding pocket. In addition, previous studies suggest that the height of the Lipid II headgroup is $19 \AA$ (Ganchev et al., 2006), and the binding pocket of this model was measured to be $15 \AA$. Since the Lipid II headgroup is flexible and the binding site provides enough room for the substrate to bend, these measurements are consistent. Together with our findings, this strongly supports the identification of this cavity as the Lipid II binding site and suggests that the Lipid II binds to MurM in an orientation similar to that of $W$. viridescens FemX binding to its substrate.

Alanyl-phosphatidylglycerol synthase (PDB: 4v34), similarly to MurM, also utilizes both lipid and alanyl-tRNA ${ }^{\text {Ala }}$ substrates $(\mathrm{He}-$ becker et al., 2015). To successfully bring these two substrates together for catalysis it possesses two binding sites, located on opposite sides of the protein, which are connected by a channel. The protein itself provides a barrier between the hydrophobic lipid and the hydrophilic tRNA, such that they do not come into close proximity with each other. The negatively charged surface patch identified previously (Fiser et al., 2003) remains present on this homology model of MurM and is located on the opposite side of the protein with respect to the Lipid II binding site, which is located within a positively charged surface patch. This negative patch is unsuitable for the binding of negatively charged tRNA, so it is unlikely that MurM shares the same mechanism of action as alanyl-phosphatidylglycerol synthase. The negatively charged surface patch may, however, be important for protein-protein interactions occurring either at the cell surface or in the cytoplasm.

These modeling studies reveal that the stem peptide protrudes perpendicular to the surface of the membrane into the active site of MurM. Therefore, in order for alanyl-tRNA ${ }^{\text {Ala }}$ to simultaneously interact with MurM, while it is located over its lipid substrate the highly negatively charged hydrophilic tRNA would have to be brought into close proximity with the negatively charged phospholipid headgroups and/or the hydrophobic phospholipid tails below them. Given that this would be a highly unfavorable interaction, we propose an alternative "ping-pong" mechanism of action for MurM whereby MurM is initially aminoacylated by alanyl- or seryl-tRNA in the cytoplasm before translocation to the cell membrane for aminoacyl transfer to Lipid II. While the MurM is in the cytoplasm and not interacting with the membrane, the positively charged patch, of the proposed Lipid II binding site, may facilitate interaction with a polyanionic substrate such as tRNA. Once this has occurred, subsequent interaction of the aminoacyl-MurM with the surface of the membrane could accommodate the correct and catalytically productive interaction of aminoacylated-MurM with Lipid II. Although this proposed mechanism is at variance with the sequential mechanism of catalysis proposed for $W$. viridescens FemX 


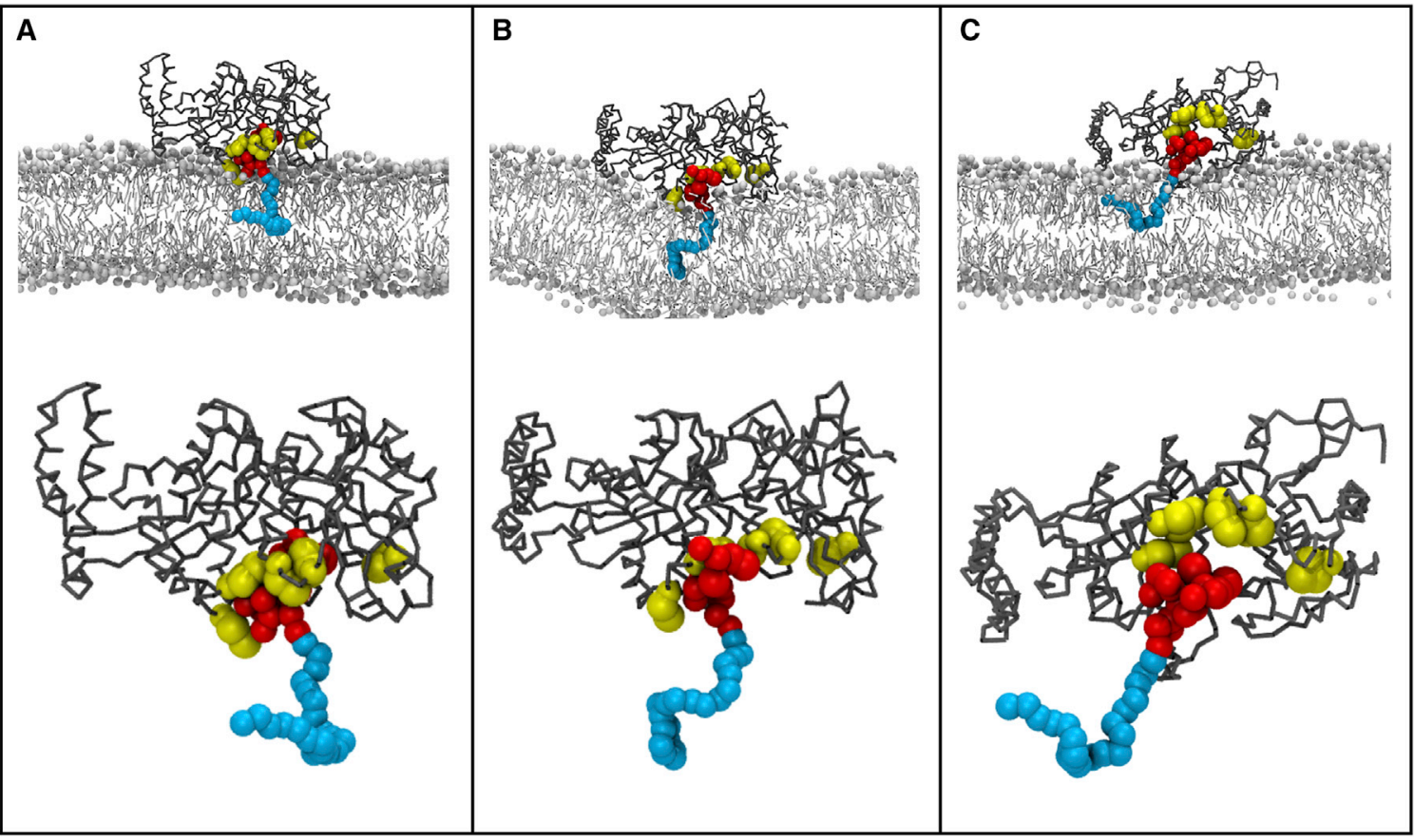

Figure 4. Lipid II binding to the putative MurM binding site

(A), (B), and (C) show the three simulations whereby Lipid II was found to bind in the putative MurM binding site. Each panel shows the MurM binding to Lipid II with respect to the membrane (top), and an enlarged image of the MurM binding to Lipid II, with the membrane removed (bottom). MurM binding site residues K35, W38, R215, and Y219 are depicted in yellow; the Lipid II headgroup and prenyl chain are rendered in red and blue, respectively.

See also Figure S1.

(Hegde and Blanchard, 2003), here both substrates are highly hydrophilic nucleotide or polynucleotide derivatives in the same cellular subcompartment and are therefore without biophysical impediment with regard to their proximity during catalysis. Here, with regard to MurM, the chemical properties and location of both substrates indicate an advantage to a mechanism that avoids their simultaneous binding.

The pneumococcal peptidoglycan is heterogeneous with respect to its composition of directly and indirectly crosslinked stem peptides. It remains unclear as to whether the activity of
MurM, and therefore the generation of indirect crosslinks, is distributed equally around the entire cell surface or whether it is localized to specific sites. Phospholipids are known to be involved in the spatial and temporal biochemistry of cells (Lin et al., 2019), and cardiolipin was shown to be enriched at the poles and septa of Escherichia coli and Bacillus subtilis, localizing specific membrane-associated proteins to these regions (Bramkamp and Lopez, 2015). Our simulations indicate that, while cardiolipin enrichment occurs within the membrane in the presence of MurM, this phospholipid is not essential for

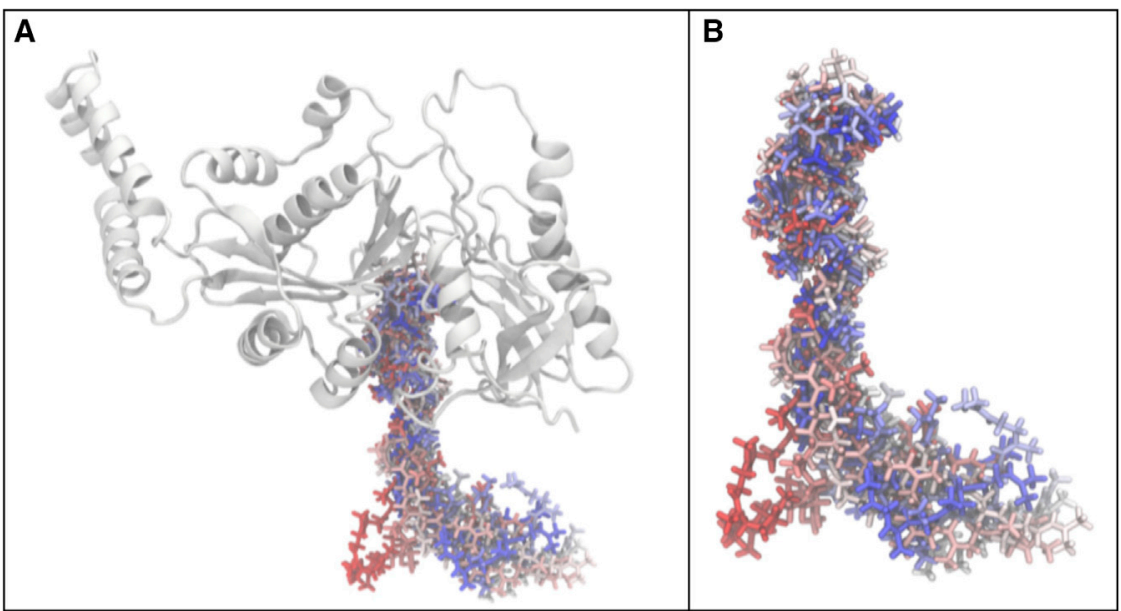

Figure 5. Different conformations of Lipid II inside MurM binding site

(A and B) MurM (gray) with Lipid II binding, colored on a blue to white to red scale with respect to simulation time, in system 5 (Table S2). 


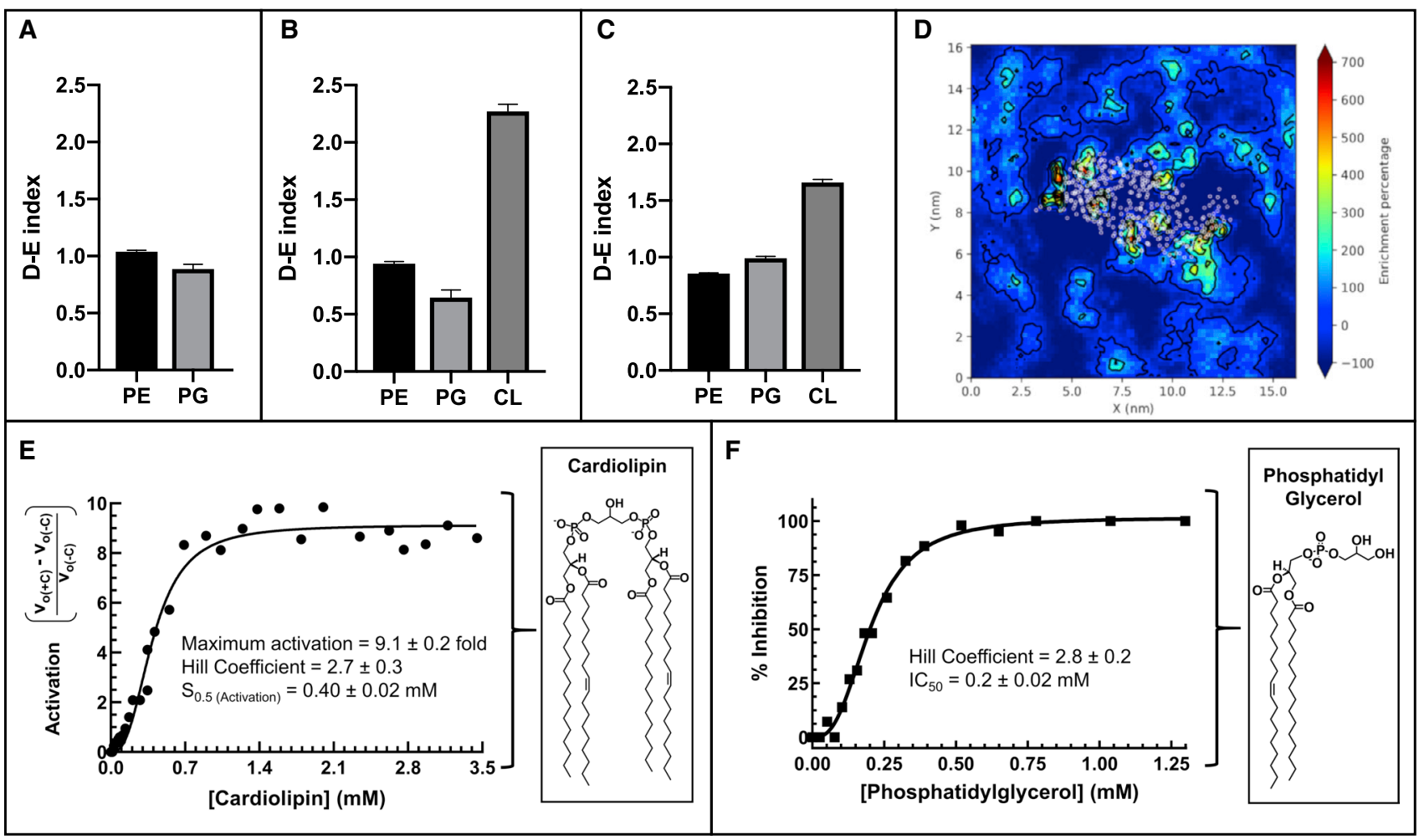

Figure 6. Interactions between MurM and membrane phospholipids

(A-C) Depletion-enrichment (D-E) indices for phosphatidylethanolamine (PE), phosphatidylglycerol (PG), and cardiolipin (CL) occurring within a 1.1-nm perimeter of the MurM protein for (A) Systems 4 and 5 (molar ratio of 75\% phosphatidylethanolamine and 25\% phosphatidylglycerol), (B) Systems 6 and 7 (molar ratio of $76 \%$ phosphatidylethanolamine, $16 \%$ phosphatidylglycerol, and $8 \%$ cardiolipin), and (C) Systems 8 and 9 (molar ratio of $72 \%$ phosphatidylethanolamine, $12 \%$ phosphatidylglycerol, and $16 \%$ cardiolipin). The D-E index was determined from 150 to $250 \mathrm{~ns}$ in 50 -ns blocks for all repeats for a total of eight values per plot. (D) Example of a D-E map with MurM at the membrane. White dots represent the center of geometry of each protein amino acid residue, and the percentage enrichment of phospholipid is indicated by the color.

(E) Activation of MurM was calculated as the product of subtraction of MurM velocity in the absence of cardiolipin ( $\left.\mathrm{V}_{0(-\mathrm{C})}\right)$ from MurM velocity in the presence of cardiolipin $\left(\mathrm{V}_{\mathrm{O}(+\mathrm{C})}\right)$ divided by $\mathrm{V}_{\mathrm{O}(-\mathrm{C})}$ and was plotted versus cardiolipin concentration.

$(\mathrm{F})$ Inhibition of MurM was calculated as $\left[\left(\mathrm{V}_{\mathrm{O}(-\mathrm{PhG})}\right)-\left(\mathrm{V}_{\mathrm{O}(+\mathrm{PhG})}\right)\right] / \mathrm{v}_{0(-\mathrm{PhG})} \times 100$ (where PhG denotes phosphatidylglycerol) and was plotted versus phosphatidylglycerol concentration. Data were fitted as described in the text. GraphPad Prism (version 8.4.1) and Matplotlib (version 3.0.3) were used for data analysis and figure preparation.

See also Figure S2.

membrane association of MurM to occur. Therefore, it remains uncertain as to whether in vivo cardiolipin is highly concentrated in patches in the membrane and is used to recruit MurM to that location or whether association of MurM with the membrane drives the enrichment of cardiolipin in the membrane.

Despite this uncertainty, we show that cardiolipin stimulates the enzymatic activity of MurM, and while it is not clear if this increased activity is a result of a direct effect on the protein or the Lipid II substrate, or both, the spatial association of cardiolipin to the MurM protein suggests that at least some of this effect may be due to direct interactions with the MurM protein.

Cardiolipin has previously been found to bind to and activate a wide range of proteins including MurG (van den Brink-van der Laan et al., 2003), rat liver protein kinase $N$ (Morrice et al., 1994; Peng et al., 1996), porcine heart AMP deaminase (Purzycka-Preis and Zydowo, 1987), rat liver multi-catalytic proteinase (Ruiz de Mena et al., 1993), E. coli glycerol-3-phosphate acyltransferase (Scheideler and Bell, 1989), E. coli dnaA (Sekimizu and Kornberg, 1988), and streptococcal hyaluronan syn- thases (Tlapak-Simmons et al., 1999a, 1999b, 2004; Weigel et al., 2006; Tlapak-Simmons et al., 1998). This further supports the contention that cardiolipin affects MurM activity by directly interacting with MurM. Similar cardiolipin-mediated sigmoidal stimulatory effects have been seen with other streptococcal membrane proteins such as the hyaluronan synthases from Streptococcus pyogenes and Streptococcus equisimilis (Tlapak-Simmons et al., 1999a, 1999b, 2004; Weigel et al., 2006). In these examples, up to 16 cardiolipin molecules are believed to associate with single hyaluronan synthase molecule (TlapakSimmons et al., 1998).

We also show that phosphatidylglycerol inhibits the catalytic activity of MurM and that the concentration of this lipid in the membrane environment surrounding the MurM changes very little. Therefore, the inhibitory effect of phosphatidylglycerol may be exerted by altering the presentation of the Lipid II substrate to MurM rather than by having a direct effect on the protein itself. It is possible that in S. pneumoniae, as in E. coli and B. subtilis, cardiolipin gathers in specific regions of the membrane, where 
in the pneumococcus it localizes and upregulates the activity of MurM, resulting in higher levels of indirect crosslinking in these regions.

While MurM alone is not sufficient for penicillin resistance, the enzyme is crucial together with mosaic S. pneumoniae PBPs for the generation of a highly resistant phenotype. Deletion of murM from resistant strains resulted in a virtual abolition of penicillin resistance that could not be restored by PBP DNA. Indeed, additional murM DNA from a resistant strain was required for full expression of donor-level penicillin resistance (Filipe and Tomasz, 2000; Smith and Klugman, 2001). Given the importance of MurM for penicillin resistance, the enrichment of cardiolipin at the MurM:membrane interface which activates MurM, and the inhibition of MurM activity by phosphatidylglycerol may regulate the penicillin resistance phenotype imparted by MurM activity, which may therefore be regulated by cardiolipin synthase activity. These findings have therefore revealed a crucial and hitherto unexplored facet of penicillin resistance suggesting the involvement of other areas of pneumococcal metabolism in the expression of clinical antibiotic resistance.

\section{Conclusions}

The MurM structural model presented in this work allowed identification of the Lipid II binding site and the contextual presentation of this substrate to MurM. Furthermore, this work characterized the impact of membrane phospholipids on MurM at the MurM:membrane interface and may have spatial mechanistic implications for the catalytic activity of this protein. MD enabled the in silico investigation into MurM-membrane interactions, which are often overlooked when studying enzymes that act at the cytoplasmic membrane interface. The subsequent in vitro experiments on the importance of phospholipids for MurM activity corroborate the in silico findings, supporting the role of phospholipids as an important contributor to the regulation of MurM at the membrane. These studies provide insights into the structure of MurM, which may guide future mutational studies and allow a more detailed analysis of the structure-function relationship of this protein. This research contributes important findings toward achieving a more complete understanding of the role of MurM in pneumococcal penicillin resistance mechanisms.

\section{STAR $\star$ METHODS}

Detailed methods are provided in the online version of this paper and include the following:

- KEY RESOURCES TABLE

- RESOURCE AVAILABILITY

- Lead contact

- Materials availability

Data and code availability

- EXPERIMENTAL MODEL AND SUBJECT DETAILS

- METHOD DETAILS

O Cloning, overexpression and purification of $S$. aureus FemX

- FemX crystallisation and data collection

$\bigcirc$ Cloning, Overexpression and Purification of S. pneumoniae(159) MurM
O Cloning, Overexpression and Purification of S. pneumoniae(Pn16) AlaRS

O Preparation of MurM substrates

S. pneumoniae MurM enzymology

O Computational studies overview

O Homology modelling of MurM

O Molecular docking of truncated Lipid II to MurM

O Molecular docking of Lipid II, Cardiolipin, Phosphatidylglycerol and Phosphatidylethanolamine to the putative MurM binding site.

○ Coarse-grained molecular dynamics simulations

O All-atom molecular dynamics simulations

- QUATIFICATION AND STATISTICAL ANALYSIS

\section{SUPPLEMENTAL INFORMATION}

Supplemental information can be found online at https://doi.org/10.1016/j.str. 2021.03.001.

\section{ACKNOWLEDGMENTS}

This research was supported in part by the Midlands Integrative Biosciences Training Partnership (MIBTP) BBSRC grant BB/J014532/1, and the Center for Doctoral Training in Theory and Modeling in Chemical Sciences (TMCS DTC) EPSRC grant EP/L015722/1, as well as MRC grants G1100127, G0400848, MR/N002679/1 and BBSRC grant BB/N003241/1. The authors would like to acknowledge the help of the Media Preparation Facility in the School of Life Sciences at the University of Warwick. We would also like to thank Dr Allister Crow for his help and support with using PyMOL.

\section{AUTHOR CONTRIBUTIONS}

Conceptualization, A.Y., A.J.L., D.I.R., and S.K.; Investigation, K.F., K.J.H., V.F., A.J.L., A.Y., J.S., and C.I.d.G.; Methodology/software/formal analysis, C.I.d.G., J.S., A.Y., and S.K.; Writing - original draft, A.Y., J.S., and C.I.d.G.; Writing - review \& editing, A.Y., A.J.L., S.K., D.I.R., and C.G.D.; Funding acquisition, A.Y., J.S., and C.G.D.; Supervision, A.J.L., D.I.R., S.K., and C.G.D.; Project administration, A.Y.; Visualization, A.Y., A.J.L., J.S., and S.K.

\section{DECLARATION OF INTERESTS}

The authors declare no competing interests.

Received: June 20, 2020

Revised: January 13, 2021

Accepted: March 1, 2021

Published: March 18, 2021

\section{REFERENCES}

Abraham, M.J., Murtola, T., Schulz, R., Páll, S., Smith, J.C., Hess, B., and Lindahl, E. (2015). GROMACS: high performance molecular simulations through multi-level parallelism from laptops to supercomputers. SoftwareX 1, 19-25.

Benson, T.E., Prince, D.B., Mutchler, V.T., Curry, K.A., Ho, A.M., Sarver, R.W., Hagadorn, J.C., Choi, G.H., and Garlick, R.L. (2002). X-ray crystal structure of Staphylococcus aureus FemA. Structure 10, 1107-1115.

Berendsen, H.J., Postma, J.V., van Gunsteren, W.F., DiNola, A., and Haak, J. (1984). Molecular dynamics with coupling to an external bath. J. Chem. Phys. 81, 3684-3690.

Biarrotte-Sorin, S., Maillard, A.P., Delettré, J., Sougakoff, W., Arthur, M., and Mayer, C. (2004). Crystal structures of Weissella viridescens FemX and its complex with UDP-MurNAc-pentapeptide: insights into FemABX family substrates recognition. Structure 12, 257-267.

Bramkamp, M., and Lopez, D. (2015). Exploring the existence of lipid rafts in bacteria. Microbiol. Mol. Biol. Rev. 79, 81-100. 
Brünger, A.T. (1992). Free R value: a novel statistical quantity for assessing the accuracy of crystal structures. Nature 355, 472-475.

Bugg, T.D.H., Braddick, D., Dowson, C.G., and Roper, D.I. (2011). Bacterial cell wall assembly: still an attractive antibacterial target. Trends Biotechnol. 29, 167-173.

Corradi, V., Mendez-Villuendas, E., Ingólfsson, H.I., Gu, R.-X., Siuda, I., Melo, M.N., Moussatova, A., DeGagné, L.J., Sejdiu, B.I., Singh, G., et al. (2018). Lipid-protein interactions are unique fingerprints for membrane proteins. ACS Cent. Sci. 4, 709-717.

Cressina, E., Lloyd, A.J., De Pascale, G., Roper, D.I., Dowson, C.G., and Bugg, T.D.H. (2007). Adenosine phosphonate inhibitors of lipid II: alanyl tRNA ligase MurM from Streptococcus pneumoniae. Bioorg. Med. Chem. Lett. 17, 4654-4656.

Cressina, E., Lloyd, A.J., De Pascale, G., Mok, B.J., Caddick, S., Roper, D.I., Dowson, C.G., and Bugg, T.D.H. (2009). Inhibition of tRNA-dependent ligase MurM from Streptococcus pneumoniae by phosphonate and sulfonamide inhibitors. Bioorg. Med. Chem. 17, 3443-3455.

Cruickshank, D. (1999). Remarks about protein structure precision. Acta Crystallogr. D Biol. Crystallogr. 55, 583-601.

Darden, T., York, D., and Pedersen, L. (1993). Particle mesh Ewald: an N log (N) method for Ewald sums in large systems. J. Chem. Phys. 98, 10089-10092.

de Jong, D.H., Singh, G., Bennett, W.D., Arnarez, C., Wassenaar, T.A., Schäfer, L.V., Periole, X., Tieleman, D.P., and Marrink, S.J. (2012). Improved parameters for the martini coarse-grained protein force field. J. Chem. Theor. Comput. 9, 687-697.

de Jong, D.H., Singh, G., Bennett, W.F., Arnarez, C., Wassenaar, T.A., Schäfer, L.V., Periole, X., Tieleman, D.P., and Marrink, S.J. (2013). Improved parameters for the martini coarse-grained protein force field. J Chem Theory Comput. 9, 687-697.

Dong, G.Q., Fan, H., Schneidman-Duhovny, D., Webb, B., and Šali, A. (2013). Optimized atomic statistical potentials: assessment of protein interfaces and loops. Bioinformatics 29, 3158-3166.

Doublié, S. (1997). Preparation of selenomethionyl proteins for phase determination. Methods Enzymol. 276, 523-530.

Emsley, P., Lohkamp, B., Scott, W.G., and Cowtan, K. (2010). Features and development of Coot. Acta Crystallogr. D Biol. Crystallogr. 66, 486-501.

Eswar, N., Webb, B., Marti-Renom, M.A., Madhusudhan, M., Eramian, D., Shen, M.-Y., Pieper, U., and Šali, A. (2006). Comparative protein structure modeling using Modeller. Curr. Protoc. Bioinformatics 15, 5-6.

Figueiredo, T.A., Sobral, R.G., Ludovice, A.M., de Almeida, J.M.F., Bui, N.K., Vollmer, W., de Lencastre, H., and Tomasz, A. (2012). Identification of genetic determinants and enzymes involved with the amidation of glutamic acid residues in the peptidoglycan of Staphylococcus aureus. PLoS Pathog. 8, e1002508.

Filipe, S.R., and Tomasz, A. (2000). Inhibition of the expression of penicillin resistance in Streptococcus pneumoniae by inactivation of cell wall muropeptide branching genes. Proc. Natl. Acad. Sci. U S A 97, 4891-4896.

Filipe, S.R., Pinho, M.G., and Tomasz, A. (2000). Characterization of the murMN operon involved in the synthesis of branched peptidoglycan peptides in Streptococcus pneumoniae. J. Biol. Chem. 275, 27768-27774.

Filipe, S.R., Severina, E., and Tomasz, A. (2001). The role of murMN operon in penicillin resistance and antibiotic tolerance of Streptococcus pneumoniae. Microb. Drug Resist. 7, 303-316.

Fiser, A., Do, R.K.G., and Šali, A. (2000). Modeling of loops in protein structures. Protein Sci. 9, 1753-1773.

Fiser, A., Filipe, S.R., and Tomasz, A. (2003). Cell wall branches, penicillin resistance and the secrets of the MurM protein. Trends Microbiol. 11, 547-553.

Fonvielle, M., Li de La Sierra-Gallay, I., El-Sagheer, A.H., Lecerf, M., Patin, D., Mellal, D., Mayer, C., Blanot, D., Gale, N., Brown, T., et al. (2013). The structure of FemXWv in complex with a peptidyl-RNA conjugate: mechanism of aminoacyl transfer from Ala-tRNAAla to peptidoglycan precursors. Angew. Chem. Int. Ed. Engl. 125, 7419-7422.
Ganchev, D., Hasper, H., Breukink, E., and de Kruijff, B. (2006). Size and orientation of the lipid II headgroup as revealed by AFM imaging. Biochemistry 45, 6195-6202.

Garcia-Bustos, J., and Tomasz, A. (1990). A biological price of antibiotic resistance: major changes in the peptidoglycan structure of penicillin-resistant pneumococci. Proc. Natl. Acad. Sci. U S A 87, 5415-5419.

Garcia-Bustos, J., Chait, B.T., and Tomasz, A. (1987). Structure of the peptide network of pneumococcal peptidoglycan. J. Biol. Chem. 262, 15400-15405.

Gowers, R.J., Linke, M., Barnoud, J., Reddy, T.J., Melo, M.N., Seyler, S.L., Domański, J., Dotson, D.L., Buchoux, S., Kenney, I.M., et al. (2016). MDAnalysis: a Python package for the rapid analysis of molecular dynamics simulations. In Proceedings of the 15th Python in Science Conference (SCIPY 2016), S. Benthall and S. Rostrup, eds. (SciPy). https://doi.org/10 25080/Majora-629e541a-00e.

Graham, J.A., Essex, J.W., and Khalid, S. (2017). PyCGTOOL: automated generation of coarse-grained molecular dynamics models from atomistic trajectories. J. Chem. Inf. Model. 57, 650-656.

Hanwell, M.D., Curtis, D.E., Lnie, D.C., Vandermeersch, T., Zurek, E., and Hutchison, G.R. (2012). Avogadro: an advanced semantic chemical editor, visualization, and analysis platform. J. Cheminform. 4, 17.

Hebecker, S., Krausze, J., Hasenkampf, T., Schneider, J., Groenewold, M., Reichelt, J., Jahn, D., Heinz, D.W., and Moser, J. (2015). Structures of two bacterial resistance factors mediating tRNA-dependent aminoacylation of phosphatidylglycerol with lysine or alanine. Proc. Natl. Acad. Sci. U S A 112, 10691-10696.

Hegde, S.S., and Blanchard, J.S. (2003). Kinetic and mechanistic characterization of recombinant Lactobacillus viridescens FemX (UDP-N-acetylmuramoyl pentapeptide-lysine N6-alanyltransferase). J. Biol. Chem. 278, 22861-22867.

Heuckeroth, R.O., Glaser, L., and Gordon, J.I. (1988). Heteroatom-substituted fatty acid analogs as substrates for $\mathrm{N}$-myristoyltransferase: an approach for studying both the enzymology and function of protein acylation. Proc. Natl. Acad. Sci. U S A 85, 8795-8799.

Huang, J., Rauscher, S., Nawrocki, G., Ran, T., Feig, M., de Groot, B.L. Grubmüller, H., and MacKerell, A.D., Jr. (2017). CHARMM36m: an improved force field for folded and intrinsically disordered proteins. Nat. Methods 14, 71-73.

Humphrey, W., Dalke, A., and Schulten, K. (1996). VMD: visual molecular dynamics. J. Mol. Graph. 14, 33-38.

Jo, S., Kim, T., Iyer, V.G., and Im, W. (2008). CHARMM-GUI: a web-based graphical user interface for CHARMM. J. Comput. Chem. 29, 1859-1865.

Jo, S., Cheng, X., Lee, J., Kim, S., Park, S.J., Patel, D.S., Beaven, A.H., Lee, K.I., Rui, H., Park, S., et al. (2017). CHARMM-GUI 10 years for biomolecular modeling and simulation. J. Comput. Chem. 38, 1114-1124.

Kabsch, W. (2010). XDS. Acta Crystallogr. D Biol. Crystallogr. 66, 125-132.

Langer, G., Cohen, S.X., Lamzin, V.S., and Perrakis, A. (2008). Automated macromolecular model building for X-ray crystallography using ARP/WARP version 7. Nat. Protoc 3, 1171.

Leahy, D.J., Hendrickson, W.A., Aukhil, I., and Erickson, H.P. (1992). Structure of a fibronectin type III domain from tenascin phased by MAD analysis of the selenomethionyl protein. Science 258, 987-991.

Lin, T.Y., Gross, W.S., Auer, G.K., and Weibel, D.B. (2019). Cardiolipin alters Rhodobacter sphaeroides cell shape by affecting peptidoglycan precursor biosynthesis. mBio 10, e02401-e02418.

Lloyd, A.J., Gilbey, A.M., Blewett, A.M., De Pascale, G., El Zoeiby, A. Levesque, R.C., Catherwood, A.C., Tomasz, A., Bugg, T.D., Roper, D.I., et al. (2008). Characterization of tRNA-dependent peptide bond formation by MurM in the synthesis of Streptococcus pneumoniae peptidoglycan. J. Biol. Chem. 283, 6402-6417.

Malde, A.K., Zuo, L., Breeze, M., Stroet, M., Poger, D., Nair, P.C., Oostenbrink, C., and Mark, A.E. (2011). An automated force field topology builder (ATB) and repository: version 1.0. J. Chem. Theor. Comput. 7, 4026-4037. 
Martí-Renom, M.A., Stuart, A.C., Fiser, A., Sánchez, R., Melo, F., and Šali, A. (2000). Comparative protein structure modeling of genes and genomes. Annu. Rev. Biophys. Biomol. Struct. 29, 291-325.

Matsuhashi, M., Dietrich, C.P., and Strominger, J.L. (1967). Biosynthesis of the peptidoglycan of bacterial cell walls III. The role of soluble ribonucleic acid and of lipid intermediates in Glycine incorporation in Staphylococcus aureus. J. Biol. Chem. 242, 3191-3206.

Morlot, C., Straume, D., Peters, K., Hegnar, O.A., Simon, N., Villard, A.M., Contreras-Martel, C., Leisico, F., Breukink, E., Gravier-Pelletier, C., et al. (2018). Structure of the essential peptidoglycan amidotransferase MurT/GatD complex from Streptococcus pneumoniae. Nat. Commun. 9. 3180.

Morrice, N.A., Gabrielli, B., Kemp, B.E., and Wettenhall, R. (1994). A cardiolipin-activated protein kinase from rat liver structurally distinct from the protein kinases C. J. Biol. Chem. 269, 20040-20046.

Morris, G.M., Huey, R., Lindstrom, W., Sanner, M.F., Belew, R.K., Goodsell, D.S., and Olson, A.J. (2009). Autodock4 and AutoDockTools4: automated docking with selective receptor flexibility. J. Comput. Chem. 16, 2785-2791.

Münch, D., Roemer, T., Lee, S.H., Engeser, M., Sahl, H.G., and Schneider, T. (2012). Identification and in vitro analysis of the GatD/MurT enzyme-complex catalyzing lipid II amidation in Staphylococcus aureus. PLoS Pathog. 8, e1002509.

Parrinello, M., and Rahman, A. (1981). Polymorphic transitions in single crystals: a new molecular dynamics method. J. Appl. Phys. 52, 7182-7190.

Peng, B., Morrice, N.A., Groenen, L.C., and Wettenhall, R.E. (1996). Phosphorylation events associated with different states of activation of a hepatic cardiolipin/protease-activated protein kinase structural identity to the protein kinase $\mathrm{N}$-type protein kinases. J. Biol. Chem. 271, 32233-32240.

Periole, X., Cavalli, M., Marrink, S.J., and Ceruso, M.A. (2009). Combining an elastic network with a coarse-grained molecular force field: structure, dynamics, and intermolecular recognition. J. Chem. Theor. Comput. 5, 2531-2543.

Pesakhov, S., Benisty, R., Sikron, N., Cohen, Z., Gomelsky, P., KhozinGoldberg, I., Dagan, R., and Porat, N. (2007). Effect of hydrogen peroxide production and the Fenton reaction on membrane composition of Streptococcus pneumoniae. Biochim. Biophys. Acta 1768, 590-597.

Pettersen, E.F., Goddard, T.D., Huang, C.C., Couch, G.S., Greenblatt, D.M., Meng, E.C., and Ferrin, T.E. (2004). UCSF Chimera-a visualization system for exploratory research and analysis. J. Comput. Chem. 25, 1605-1612.

Purzycka-Preis, J., and Zydowo, M. (1987). Regulatory effect of pig heart phospholipids on heart muscle AMP-deaminase. Int. J. Biochem. 19, 565-568.

Ruiz de Mena, I., Mahillo, E., Arribas, J., and Castano, J. (1993). Kinetic mechanism of activation by cardiolipin (diphosphatidylglycerol) of the rat liver multicatalytic proteinase. Biochem. J. 296, 93-97.

Sali, A., and Blundell, T.L. (1993). Comparative protein modelling by satisfaction of spatial restraints. J. Mol. Biol. 234, 779-815.

Scheideler, M., and Bell, R.M. (1989). Phospholipid dependence of homogeneous, reconstituted sn-glycerol-3-phosphate acyltransferase of Escherichia coli. J. Biol. Chem. 264, 12455-12461.

Schlame, M. (2008). Thematic Review Series: glycerolipids. Cardiolipin synthesis for the assembly of bacterial and mitochondrial membranes. J. Lipid Res. 49, 1607-1620.

Schneider, T., and Sahl, H.G. (2010). An oldie but a goodie-cell wall biosynthesis as antibiotic target pathway. Int. J. Med. Microbiol. 300, 161-169.

Schneider, T., Senn, M.M., Berger-Bächi, B., Tossi, A., Sahl, H.G., and Wiedemann, I. (2004). In vitro assembly of a complete, pentaglycine interpeptide bridge containing cell wall precursor (lipid II-Gly5) of Staphylococcus aureus. Mol. Microbiol. 53, 675-685.

Sekimizu, K., and Kornberg, A. (1988). Cardiolipin activation of dnaA protein, the initiation protein of replication in Escherichia coli. J. Biol. Chem. 263, $7131-7135$.
Severin, A., and Tomasz, A. (1996). Naturally occurring peptidoglycan variants of Streptococcus pneumoniae. J. Bacteriol. 178, 168-174.

Shearer, J., Jefferies, D., and Khalid, S. (2019). Outer membrane proteins OmpA, FhuA, OmpF, EstA, BtuB, and OmpX have unique lipopolysaccharide fingerprints. J. Chem. Theor. Comput. 15, 2608-2619.

Sheldrick, G.M. (2010). Experimental phasing with SHELXC/D/E: combining chain tracing with density modification. Acta Crystallogr. D Biol. Crystallogr. $66,479-485$.

Shen, M.Y., and Sali, A. (2006). Statistical potential for assessment and prediction of protein structures. Protein Sci. 15, 2507-2524.

Sievers, F., Wilm, A., Dineen, D., Gibson, T.J., Karplus, K., Li, W., Lopez, R., McWilliam, H., Remmert, M., Söding, J., et al. (2011). Fast, scalable generation of high-quality protein multiple sequence alignments using Clustal Omega. Mol. Syst. Biol. 7, 539.

Smith, A.M., and Klugman, K.P. (2001). Alterations in MurM, a cell wall muropeptide branching enzyme, increase high-level penicillin and cephalosporin resistance in Streptococcus pneumoniae. Antimicrob. Agents Chemother. 45, 2393-2396.

Smith, J.M., Dowson, C.G., and Spratt, B.G. (1991). Localized sex in bacteria. Nature 349, 29-31.

Tironi, I.G., Sperb, R., Smith, P.E., and van Gunsteren, W.F. (1995). A generalized reaction field method for molecular dynamics simulations. J. Chem. Phys. 102, 5451-5459.

Tlapak-Simmons, V.L., Kempner, E.S., Baggenstoss, B.A., and Weigel, P.H. (1998). The active streptococcal hyaluronan synthases (HASs) contain a single HAS monomer and multiple cardiolipin molecules. J. Biol. Chem. 273, 26100-26109.

Tlapak-Simmons, V.L., Baggenstoss, B.A., Clyne, T., and Weigel, P.H. (1999a). Purification and lipid dependence of the recombinant hyaluronan synthases from Streptococcus pyogenes and Streptococcus equisimilis. J. Biol. Chem. 274, 4239-4245.

Tlapak-Simmons, V.L., Baggenstoss, B.A., Kumari, K., Heldermon, C., and Weigel, P.H. (1999b). Kinetic characterization of the recombinant hyaluronan synthases from Streptococcus pyogenes and Streptococcus equisimilis. J. Biol. Chem. 274, 4246-4253.

Tlapak-Simmons, V.L., Baron, C.A., and Weigel, P.H. (2004). Characterization of the purified hyaluronan synthase from Streptococcus equisimilis Biochemistry 43, 9234-9242.

Trombe, M.C., Lanéelle, M.A., and Lanéelle, G. (1979). Lipid composition of aminopterin-resistant and sensitive strains of Streptococcus pneumoniae. Effect of aminopterin inhibition. Biochim. Biophys. Acta 574, 290-300.

Trott, O., and Olson, A.J. (2010). AutoDock Vina: improving the speed and accuracy of docking with a new scoring function, efficient optimization, and multithreading. J. Comput. Chem. 31, 455-461.

Vagin, A.A., Steiner, R.A., Lebedev, A.A., Potterton, L., McNicholas, S., Long, F., and Murshudov, G.N. (2004). REFMAC5 dictionary: organization of prior chemical knowledge and guidelines for its use. Acta Crystallogr. D Biol. Crystallogr. 60, 2184-2195.

van den Brink-van der Laan, E., Boots, J.-W.P., Spelbrink, R.E., Kool, G.M. Breukink, E., Killian, J.A., and de Kruijff, B. (2003). Membrane interaction of the glycosyl-transferase MurG: a special role for cardiolipin. J. Bacteriol. 185, 3773-3779.

Van Zundert, G., Rodrigues, J., Trellet, M., Schmitz, C., Kastritis, P., Karaca, E., Melquiond, A., vanDijk, M., De Vries, S., and Bonvin, A. (2016). The haddock2.2 web server: user-friendly integrative modeling of biomolecular complexes. J. Mol. Biol. 428, 720-725.

Wang, J., Wolf, R.M., Caldwell, J.W., Kollman, P.A., and Case, D.A. (2004). Development and testing of a general amber force field. J. Comput. Chem. 25, 1157-1174.

Wassenaar, T.A., Pluhackova, K., Bockmann, R.A., Marrink, S.J., and Tieleman, D.P. (2014). Going backward: a flexible geometric approach to reverse transformation from coarse grained to atomistic models. J. Chem. Theor. Comput. 10, 676-690. 
Weigel, P.H., Kyossev, Z., and Torres, L.C. (2006). Phospholipid dependence and liposome reconstitution of purified hyaluronan synthase. J. Biol. Chem. 281, 36542-36551.

Williams, C.J., Headd, J.J., Moriarty, N.W., Prisant, M.G., Videau, L.L., Deis, L.N., Verma, V., Keedy, D.A., Hintze, B.J., Chen, V.B., et al. (2008). MolProbity: more and better reference data for improved all-atom structure validation. Protein Sci. 27, 293-315.

Winter, G. (2010). xia2: an expert system for macromolecular crystallography data reduction. J. Appl. Cryst. 43, 186-190.

Witzke, S., Petersen, M., Carpenter, T.S., and Khalid, S. (2016). Molecular dynamics simulations reveal the conformational flexibility of lipid II and its loose association with the defensin plectasin in the Staphylococcus aureus membrane. Biochemistry 55, 3303-3314.

Wright, M.H., Heal, W.P., Mann, D.J., and Tate, E.W. (2010). Protein myristoylation in health and disease. J. Chem. Biol. 3, 19-35.

Zapun, A., Philippe, J., Abrahams, K.A., Signor, L., Roper, D.I., Breukink, E., and Vernet, T. (2013). In vitro reconstitution of peptidoglycan assembly from the Gram-positive pathogen Streptococcus pneumoniae. ACS Chem. Biol. 8, 2688-2696.

Zubay, G. (1962). The isolation and fractionation of soluble ribonucleic acid. J. Mol. Biol. 4, 347-356. 


\section{STAR $\star$ METHODS}

\section{KEY RESOURCES TABLE}

\begin{tabular}{|c|c|c|}
\hline REAGENT or RESOURCE & SOURCE & IDENTIFIER \\
\hline \multicolumn{3}{|l|}{ Bacterial and Virus Strains } \\
\hline $\begin{array}{l}\text { E. coli BL21 Star (DE3): } \mathrm{F}^{-} \text {omp T hsd } \mathrm{S}_{\mathrm{B}} \\
\left(\mathrm{r}_{\mathrm{B}}^{-} \mathrm{m}_{\mathrm{B}}^{-}\right) \text {gal dcm rne131 (DE3) }\end{array}$ & Invitrogen & Cat\# C601003 \\
\hline $\begin{array}{l}\text { E. coli B834 (DE3) - } \mathrm{F}^{-} \text {ompt hsd } \mathrm{S}_{\mathrm{B}} \\
\left(\mathrm{r}_{\mathrm{B}}^{-} \mathrm{m}_{\mathrm{B}}^{-}\right) \text {gal dcm met (DE3) }\end{array}$ & Novagen & Cat\# 69401 \\
\hline $\begin{array}{l}\text { E.coli Rosetta } 2 \text { (DE3). F- ompT hsdSB(rB- mB-) } \\
\text { gal dcm (DE3) pRARE2 (CamR) }\end{array}$ & Novagen & Cat\# 71405 \\
\hline $\begin{array}{l}\text { E. coli BL21 Star pRosetta2 (DE3) - F- ompT hsdSB } \\
\text { (rB- } m B \text {-) gal dcm rne131 pRARE (DE3) }\end{array}$ & Lloyd et al. (2008) & $\mathrm{N} / \mathrm{A}$ \\
\hline $\begin{array}{l}\text { E. coli C41 (DE3) (a derivative of BL21(DE3) [F_ampT } \\
h s d S 8 \text { (r8m8)gal dcm DE3]) pRIL (Cam }{ }^{\mathrm{R}} \text { ) }\end{array}$ & Lloyd et al. (2008) & $\mathrm{N} / \mathrm{A}$ \\
\hline $\begin{array}{l}\text { E. coli B834 (DE3): pRosetta2 - } \mathrm{F}^{-} \text {ompT hsdS } \mathrm{B}_{\mathrm{B}}\left(\mathrm{r}_{\mathrm{B}}^{-} \mathrm{m}_{\mathrm{B}}^{-}\right) \\
\text {gal dcm met pRARE2 (DE3) }\end{array}$ & Novagen & Cat\# 69041 \\
\hline M. flavus (M. luteus) (Stanley130.21) & NCIMB & Cat\# 8166 \\
\hline \multicolumn{3}{|l|}{ Chemicals, Peptides, and Recombinant Proteins } \\
\hline Poly(ethylene glycol) 8000 & Fluka & Cat\# 81272 \\
\hline L-Alanine, $[2,3-3 \mathrm{H}]$ & Moravek & Cat\# MT-886 \\
\hline Lipid II (Lys) & BACWAN/Lloyd et al. (2008) & Cat\# C55-LII-5K \\
\hline $\begin{array}{l}\text { Phosphatidylglycerol } \\
\text { L-alpha-Phosphatidyl-DL-glycerol sodium salt from } \\
\text { egg yolk lecithin, =99\% (TLC), lyophilized powder }\end{array}$ & MERCK LIFE SCIENCE UK LTD & Cat\# P8318 \\
\hline $\begin{array}{l}\text { Cardiolipin } \\
\text { Cardiolipin sodium salt from bovine heart, =98\% (TLC), } \\
\text { lyophilized powder }\end{array}$ & MERCK LIFE SCIENCE UK LTD & Cat\# C0563 \\
\hline JCSG-plus ${ }^{\mathrm{TM}}$ crystallization screen & Molecular Dimensions & Cat\# MD1-37 \\
\hline PACT premier ${ }^{\mathrm{TM}}$ crystallization screen & Molecular Dimensions & Cat\# MD1-29 \\
\hline Morpheus $₫$ crystallization screen & Molecular Dimensions & Cat\# MD1-46 \\
\hline Cobalt TALON resin & Takara & Cat\# 635502 \\
\hline Nickel- Chelating Sepharose & Cytiva & Cat\# 17526801 \\
\hline Superdex 75 Size exclusion media & Cytiva & Cat\# 17104404 \\
\hline Sephacryl S200 size exclusion media & Cytiva & Cat\# 17058401 \\
\hline Selenomethionine & Acros Organics & Cat\# 259960025 \\
\hline Tomato etch virus (TEV) Protease & New England Biolabs & Cat\# P8112 \\
\hline Bsal & New England Biolabs & Cat\# R0535S \\
\hline Xho1 & New England Biolabs & Cat\# R0146S \\
\hline Hen egg white lysozyme & MERCK LIFE SCIENCE UK LTD & Cat\# L6976 \\
\hline \multicolumn{3}{|l|}{ Deposited Data } \\
\hline S. aureus FemX & This paper & PDB: 6SNR \\
\hline \multicolumn{3}{|l|}{ Oligonucleotides } \\
\hline $\begin{array}{l}\text { FemX Forward TTTGCGGGTGGTCTCCCATGGAAA } \\
\text { AGATGCATATCACTAATCAGG }\end{array}$ & IDT DNA & $\mathrm{N} / \mathrm{A}$ \\
\hline $\begin{array}{l}\text { S. aureus FemX Reverse } \\
\text { TTIGCGCTCGAGGCCCTGAAAATACAG } \\
\text { GTITCTITCGTITAATTTACGAGATA } \\
\text { TTITAATITAGC }\end{array}$ & IDT DNA & $\mathrm{N} / \mathrm{A}$ \\
\hline
\end{tabular}




\begin{tabular}{|c|c|c|}
\hline \multicolumn{3}{|l|}{ Continued } \\
\hline REAGENT or RESOURCE & SOURCE & IDENTIFIER \\
\hline \multicolumn{3}{|l|}{ Recombinant DNA } \\
\hline $\begin{array}{l}\text { pRosetta } 2 \text { (purified from Escherichia coli } \\
\text { Rosetta }^{T M} 2 \text { (DE3)) }\end{array}$ & Novagen & Cat\# 71405 \\
\hline pET28a(+) & Novagen & Cat\# 69864 \\
\hline pET21b::MurM 159 & Lloyd et al. (2008) & $\mathrm{N} / \mathrm{A}$ \\
\hline pET26b::alaRS & Lloyd et al. (2008) & N/A \\
\hline pET28::FemX & This paper & N/A \\
\hline \multicolumn{3}{|l|}{ Software and Algorithms } \\
\hline GraphPad Prism Version 8.4.1. & $\begin{array}{l}\text { GraphPad Soft-ware, } \\
\text { San Diego, CA, USA. }\end{array}$ & www.graphpad.com \\
\hline Xia2 & Winter (2010) & https://xia2.github.io/ \\
\hline XDS package & Kabsch (2010) & http://xds.mpimf-heidelberg.mpg.de \\
\hline SHELX suite & Sheldrick (2010) & $\begin{array}{l}\text { https://www.shelxle.org/shelx/ } \\
\text { eingabe.php }\end{array}$ \\
\hline ARP/wARP & Langer et al. (2008) & https://www.embl-hamburg.de/ARP/ \\
\hline REFMAC & Vagin et al. (2004) & $\begin{array}{l}\text { https://www2.mrc-Imb.cam.ac.uk/ } \\
\text { groups/murshudov/content/refmac/ } \\
\text { refmac.html }\end{array}$ \\
\hline COOT & Emsley et al. (2010) & $\begin{array}{l}\text { https://www2.mrc-Imb.cam.ac.uk/ } \\
\text { personal/pemsley/coot/ }\end{array}$ \\
\hline MODELLER & $\begin{array}{l}\text { Eswar et al. (2006); Martí-Renom } \\
\text { et al., 2000; sali and Blundell } \\
\text { (1993); Fiser et al. (2000) }\end{array}$ & https://salilab.org/modeller/ \\
\hline Chimera (Version 1.13.1) & Pettersen et al., (2004) & https://www.cgl.ucsf.edu/chimera/ \\
\hline Discrete Optimized Protein Energy (DOPE-HR) & Shen and sali (2006) & \\
\hline Statistically Optimized Atomic Potentials (SOAP) & Dong et al. (2013) & https://github.com/salilab/SOAP \\
\hline PyMOL (Version 2.2.0) & $\begin{array}{l}\text { Schrodinger, LLC. } 2010 . \\
\text { The PyMOL Molecular } \\
\text { Graphics System, Version } 2.1 .0\end{array}$ & https://pymol.org/2/\#download \\
\hline Avogadro2 software & Hanwell et al. (2012) & $\begin{array}{l}\text { https://www.openchemistry.org/ } \\
\text { downloads/ }\end{array}$ \\
\hline AutoDock Vina & Trott and Olson (2010) & $\begin{array}{l}\text { http://vina.scripps.edu/ } \\
\text { download.html }\end{array}$ \\
\hline HADDOCK web server & Van Zundert et al. (2016) & $\begin{array}{l}\text { https://bianca.science.uu.nl/ } \\
\text { haddock2.4/ }\end{array}$ \\
\hline GROMACS package (Version 2018) & Abraham et al. (2015) & http://www.gromacs.org/ \\
\hline Auto-mated topology builder (ATB) web-interface & Malde et al. (2011) & https://atb.uq.edu.au/ \\
\hline PyCGTOOL & Graham et al. (2017) & https://pypi.org/project/pycgtool/ \\
\hline CHARMM-GUI web interface & Jo et al. (2017) & http://charmm-gui.org/ \\
\hline backward script & Wassenaar et al. (2014) & $\begin{array}{l}\text { http://www.cgmartini.nl/index.php/ } \\
\text { downloads/tools/240-backward }\end{array}$ \\
\hline Particle mesh Ewald (PME) algorithm & Darden et al. (1993) & $\mathrm{N} / \mathrm{A}$ \\
\hline Visual Molecular Dynamics (VMD) & Humphrey et al. (1996) & $\begin{array}{l}\text { https://www.ks.uiuc.edu/ } \\
\text { Research/vmd/ }\end{array}$ \\
\hline
\end{tabular}

\section{RESOURCE AVAILABILITY}

\section{Lead contact}

Further information and requests for resources and reagents should be directed to and will be fulfilled by the Lead Contact, David Roper (David.Roper@warwick.ac.uk).

\section{Materials availability}

This study did not generate any new unique reagents. Plasmids will be available by Materials Transfer Agreement (MTA) request in line with University of Warwick IP requirements. 
Data and code availability

No novel code was generated during this work. Modelling scripts and raw data are available from the authors upon request.

\section{EXPERIMENTAL MODEL AND SUBJECT DETAILS}

E. coli B834 (DE3) - F- ompT hsdSB (rB- mB-) gal dcm met (DE3), referred to as E. coli B834 (DE3) in the method details section was transformed with pRosetta2 to yield $E$. coli B834 (DE3): pRosetta2 - $\mathrm{F}^{-}$ompT hsdS $\mathrm{B}_{\mathrm{B}}\left(\mathrm{r}_{\mathrm{B}}{ }^{-} \mathrm{m}_{\mathrm{B}}{ }^{-}\right)$gal dcm met (DE3) which was employed as the expression host for selenomethionine substituted S. aureus FemX. E. coli C41 (DE3) (a derivative of BL21(DE3) [F_ampT hsdS8 $(r 8 m 8) g a l ~ d c m ~ D E 3])$ was transformed with $\left.\mathrm{pRIL}\left(\mathrm{Cam}^{\mathrm{R}}\right)\right)$. The resulting transformant referred to as $E$. coli C41 (DE3)/pRIL in the method details section was employed as the expression host for expression of S. pneumoniae (159) MurM. E. coli BL21 Star (DE3): $\mathrm{F}^{-}$ompT hsdS $\mathrm{B}_{\mathrm{B}}\left(\mathrm{r}_{\mathrm{B}}{ }^{-} \mathrm{m}_{\mathrm{B}}{ }^{-}\right) \mathrm{gal}$ dcm rne131 (DE3) was transformed with pRosetta2 to yield E. coli BL21 Star pRosetta2 (DE3) F- ompT hsdSB (rB- mB-) gal dcm rne131 (DE3) referred to as E. coli BL21(DE3) star/pRosetta2 in the methods details section which was utilized as the expression host for expression of S. pneumoniae (Pn16) alanyl-tRNA ${ }^{\text {Ala }}$ synthetase (AlaRS). Expression of selenomethionine-labelled FemX was performed in M9 media supplemented with all 19 canonical amino acids and $40 \mathrm{mg} \mathrm{mL}^{-1}$ [LA1] Lselenomethionine in place in L-methionine. Expression of the remaining proteins were performed in luria broth. For all expression experiments freshly transformed expression hosts were cultured shaken at $180-200 \mathrm{rpm}$ at $37^{\circ} \mathrm{C}$ in the presence of the appropriate antibiotics (30 mg mL ${ }^{-1}$ kanamycin, $30 \mathrm{mg} \mathrm{mL}^{-1}$ chloroamphenicol for FemX; $50 \mathrm{mg} \mathrm{mL}^{-1}$ carbenicillin, $30 \mathrm{mg} \mathrm{mL}^{-1} \mathrm{chloroamphe-}^{-}$ nicol for MurM and $30 \mathrm{mg} \mathrm{mL}^{-1}$ kanamycin, $30 \mathrm{mg} \mathrm{mL}^{-1}$ chloroamphenicol for AlaRS) until mid-exponential phase (Optical density at $\left.600 \mathrm{~nm}\left(\mathrm{OD}_{600}\right)=0.4-0.6\right)$. At this point, cultures were supplemented with fresh antibiotics and protein expression was induced by 1 $\mathrm{mM}$ isopropyl-D-1-thiogalactopyranoside at $25^{\circ} \mathrm{C}$ for $4 \mathrm{~h}$. Micrococcus flavus (Synonymous with M. luteus) was cultured shaken at $200 \mathrm{rpm}$ at $37^{\circ} \mathrm{C}$ in tryptic soy broth to late exponential phase $\left(\mathrm{OD}_{600}\right.$ of 4$)$.

\section{METHOD DETAILS}

Cloning, overexpression and purification of S. aureus FemX

The S. aureus Mu50 FemX gene was amplified from chromosomal DNA using Oligonucleotides FemX forward: TTTGCGGGTGGTCTCCCATGGAAAAGATGCATATC ACTAATCAGG and FemX Reverse: TTTGCGCTCGAGGCCCTGAAAATACAG GTTTC TITTCGTITTAATTTACGAGATATTTTAATTTTAGC. The resulting PCR fragment was cleaved with Bsal and Xhol and cloned into $\mathrm{pET} 28$ between the $\mathrm{Ncol}$ and $\mathrm{Xhol}$ restriction sites to create $\mathrm{pET} 28::$ FemX, containing a Tobacco Etch Virus (TEV) protease cleavable C-terminal hexa-histidine tag. E. coli B834 (DE3) harbouring plasmid pRosetta2 (which supplies seven rare tRNAs to support expression of genes in E. coli and derived from the Rosetta 2(DE3) strain) were transformed with pET28::FemX. Transformed E. coli B834 (DE3) pRosetta2 were used to inoculate M9 media supplemented with $30 \mathrm{mg} \mathrm{mL}^{-1}$ kanamycin, $30 \mathrm{mg} \mathrm{mL}^{-1} \mathrm{chloroam}$ phenicol, and $40 \mathrm{mg} \mathrm{mL}^{-1}$ of each of the canonical amino acids [LA1] except L-methionine, which was replaced by $40 \mathrm{mg} \mathrm{mL}^{-1}$ (Doublié, 1997). Transformants were cultured at $37^{\circ} \mathrm{C}$ at $180 \mathrm{rpm}$ until an optical density at $600 \mathrm{~nm}\left(\mathrm{OD}_{600}\right)$ of $0.4-0.6$ was reached. Protein expression was induced by $1 \mathrm{mM}$ isopropyl-D-1-thiogalactopyranoside at $25^{\circ} \mathrm{C}$ for 4 hours. Cells were harvested by centrifugation at $6,000 \mathrm{xg}$ for 15 minutes and cell pellets containing 4-6 g of cells were resuspended in $20 \mathrm{~mL}$ of $50 \mathrm{mM}$ sodium phosphate $\mathrm{pH} 7.0,1 \mathrm{M} \mathrm{NaCl}$ and $2.5 \mathrm{mg} \cdot \mathrm{mL}^{-1}$ lysozyme to which one tablet of Pierce EDTA free Protease Inhibitor was added. The cell suspension was incubated with slow rotation for 30 minutes at $4{ }^{\circ} \mathrm{C}$ before disruption using a Bandelin Sonopuls sonicator with $3 \times 30$ second bursts at $70 \%$ power. The lysate was clarified by centrifugation at $50,000 \mathrm{xg}$ at $4{ }^{\circ} \mathrm{C}$ for 30 minutes. FemX was then purified by immobilised metal affinity chromatography (IMAC) using a $5 \mathrm{~mL}$ gravity fed column of cobalt Talon resin equilibrated with $50 \mathrm{~mL}$ of $50 \mathrm{mM}$ sodium phosphate $\mathrm{pH} 7.0,500 \mathrm{mM} \mathrm{NaCl}, 20 \%(\mathrm{v} / \mathrm{v})$ glycerol (equilibration buffer) supplemented with $10 \mathrm{mM}$ imidazole. Once the 50,000 $\mathrm{xg}$ supernatant was loaded onto the column, it was eluted sequentially with $50 \mathrm{~mL}$ of equilibration buffer with $10 \mathrm{mM}$ imidazole, $30 \mathrm{~mL}$ of equilibration buffer with $50 \mathrm{mM}$ imidazole and $30 \mathrm{~mL}$ of equilibration buffer with $200 \mathrm{mM}$ imidazole. $10 \mathrm{~mL}$ Fractions were analysed by SDS-PAGE and those containing FemX were pooled and concentrated, using a vivaspin 20 centrifugal concentrator (10,000 molecular weight cut off (MWCO), as required. Size exclusion chromatography in 50 mM sodium phosphate $\mathrm{pH} 7.0,500 \mathrm{mM} \mathrm{NaCl}$ and $20 \%$ (v/v) glycerol was used to further purify FemX on a Superdex 75 10/300 column. The histidine tag was then removed from the FemX protein by digestion with histidine-tagged TEV protease at a molar ratio of 100:1 FemX : TEV protease at $4^{\circ} \mathrm{C}$ overnight. Cleaved and uncleaved protein were separated by a reverse IMAC following the procedure described above.

Fem $X$ crystallisation and data collection

FemX was exchanged into $50 \mathrm{mM}$ ethanolamine $\mathrm{pH} 10.0,100 \mathrm{mM} \mathrm{NaCl}$ and $20 \%(\mathrm{v} / \mathrm{v})$ glycerol, concentrated to $15 \mathrm{mg}^{\mathrm{mL}} \mathrm{m}^{-1}$ using a vivaspin 20 centrifugal concentrator column with a 10,000 MWCO and screened for suitable crystallisation conditions using a honeybee 963 crystallisation robot against JCSG plus, PACT primer and Morpheus crystallisation screens. Crystals obtained from the Morpheus screen were used directly for data collection experiments, although crystallization conditions were further refined to $0.12 \mathrm{M}$ ethylene glycol, $0.1 \mathrm{M} \mathrm{MES} /$ imidazole $\mathrm{pH} 6.3$ and $28 \%(\mathrm{w} / \mathrm{v})$ ethylene glycol-PEG 8000 . Crystals were frozen directly for Xray diffraction data experiments on the I04-1 beamline at the Diamond synchrotron (Didcot, UK) using a Pilatus 6M-F detector. Data were processed automatically using Xia2 (Winter, 2010) to $1.62 \AA$. Molecular replacement was not successful so selenomethionine containing FemX protein was produced and used to obtain FemX crystals (FemX-SeMet) in the same crystallisation conditions and 
the structure was solved by single anomalous diffraction (Leahy et al., 1992). X-ray data from the FemX-SeMet crystal were collected on the 102 beamline at the Diamond synchrotron (Didcot, UK) using a Pilatus $6 \mathrm{M}$ detector. All data were indexed, integrated and scaled using the XDS package (Kabsch, 2010). All 10 of expected selenium atoms in the asymmetric unit were located and refined by the SHELX suite (Sheldrick, 2010). These sites were used to obtain preliminary phases. The starting model was built by ARP/wARP (Langer et al., 2008). This model was used to refine the higher resolution data. The structure was refined using iterative cycles of REFMAC (Vagin et al., 2004) and model building/solvent addition with COOT (Emsley et al., 2010).

Cloning, Overexpression and Purification of S. pneumoniae(159) MurM

As described in Lloyd et al. (2008), the MurM allele of S. pneumoniae(159) was cloned with a C-terminal histidine tag into pET21b and over-expressed in E. coli C41 (DE3)/pRIL. Cells were harvested by centrifugation and treated with 2.5 mg. $\mathrm{mL}^{-1}$ hen egg white lysozyme prior sonication. MurM was solubilised with $1 \mathrm{M} \mathrm{NaCl}$ and fractionated between $25 \%$ and $50 \%$ of saturation ammonium sulfate followed by purification by size exclusion chromatography with Sephacryl S-200 and by immobilized metal affinity chromatography (IMAC) using cobalt Talon resin. The purity and identity of the final products of these purifications were assessed by SDS-PAGE.

Cloning, Overexpression and Purification of S. pneumoniae(Pn16) AlaRS

As described in Lloyd et al. (2008), the AlaRS allele of S. pneumoniae(Pn16) was cloned into pET26a and over-expressed in E. coli BL21(DE3) star/pRosetta2 The soluble protein was purified using nickel-chelated Chelating sepharose, desalted and further purified by anion exchange chromatography on a $0.98 \mathrm{ml} \mathrm{MonoQ}^{\mathrm{TM}}$, column. The purity and identity of the final products of these purifications were assessed by SDS-PAGE.

\section{Preparation of MurM substrates}

The substrates used for assays of MurM were prepared as follows:

Lipid II(Lys): The peptidoglycan intermediate Lipid II(Lys) (undecaprenyl pyrophosphoryl N-N-acetyl muramyl (N-acetyl glucosaminyl) L-alanyl- $\gamma$-D-glutamyl-L-lysyl-D-alanyl-D-alanine) was prepared by re-capitulation of the peptidoglycan synthesis pathway as described (Lloyd et al., 2008).

$\left[{ }^{3} \mathbf{H}\right]$-Alanyl-tRNA ${ }^{\text {Ala }}$ : Micrococcus flavus tRNA was isolated from cell pellets of $M$. flavus cultures grown to late exponential phase by phenol extraction followed by isopropanol precipitation, anion exchange chromatography and ethanol precipitation as described by Zubay (1962) as adapted by Lloyd et al. (2008). tRNAs were renatured in $2 \mathrm{mM} \mathrm{MgCl}_{2}$ at $60^{\circ} \mathrm{C}$ and aminoacylated with [2,3- $\left.{ }^{3} \mathrm{H}\right]-\mathrm{L}-$ alanine S. pneumoniae (Pn16) AlaRS as described by Lloyd et al. (2008) and quantitated by liquid scintillation counting.

\section{S. pneumoniae MurM enzymology}

MurM was assayed as described by Lloyd et al. (2008) in duplicate in a final volume of $35 \mu \mathrm{l}$ of $50 \mathrm{mM} 3-(\mathrm{N}$-morpholino)-propane sulphonic acid adjusted to $\mathrm{pH} 6.8,30 \mathrm{mM} \mathrm{KCl}, 10 \mathrm{mM} \mathrm{MgCl}_{2}, 1.5 \%$ (w/v) CHAPS (Assay Buffer), $1 \mathrm{mM} \mathrm{DTT,} 1 \mathrm{mM}$ L-alanine,

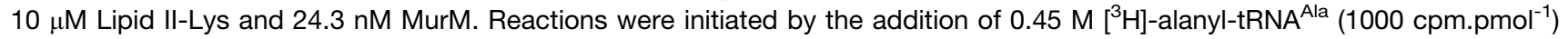
and were incubated at $37^{\circ} \mathrm{C}$ for two minutes, over which time frame, product accumulation was linear with respect to time. Where the impact of cardiolipin or phosphatidylglycerol on MurM activity was assessed, the required amounts of $10 \mathrm{mg} / \mathrm{mL}$ stocks of each phospholipid in ethanol or chloroform/methanol (49:1) were dried down in the reaction vials the assays were to be performed in, and solubilised by addition of assay buffer. Reactions were terminated by the addition of $35 \mu$ l of ice-cold $6 \mathrm{M}$ pyridinium acetate $\mathrm{pH} 4.5$ and $70 \mu \mathrm{l}$ ice-cold n-butanol. The incubations were rapidly mixed and centrifuged for 5 minutes at $1^{\circ} \mathrm{C}$ at $13,000 \mathrm{xg}$, after which time the $n$-butanol phase was washed with $70 \mu$ of water and then assayed for $\left[{ }^{3} \mathrm{H}\right]$-Lipid II-L-Ala by liquid scintillation counting. Tritium counts accumulated in control reactions performed without Lipid II(Lys) were subtracted from corresponding data acquired in the presence of this substrate. MurM activities in the presence of phospholipid were related to the activity of the enzyme in the absence of phospholipid and plotted as fold activation or percentage inhibition vs phospholipid concentration. The data were then fitted using GraphPad Prism (Version 8.4.1) to either of equations 1 or 2 as appropriate:

$$
\begin{gathered}
\text { Fold Activation }=\frac{\text { Maximum Activation } \cdot[\text { Cardiolipin }]^{h}}{S_{0.5(\text { Activation })}{ }^{h}+[\text { Cardiolipin }]^{h}} \\
\% \text { Inhibition }=\frac{100 \cdot[\text { Phosphatidylglycerol }]^{h}}{I C_{50}{ }^{h}+[\text { Phosphatidylglycerol }]^{h}}
\end{gathered}
$$

(Equation 1)

Maximum activation and $\mathrm{S}_{0.5}$ (Activation) (Equation 1) corresponded to the degree of activation at infinite cardiolipin concentration and the cardiolipin concentration required to elicit half maximal activation respectively. $I_{50}$ (Equation 2) corresponds to the phosphatidylglycerol concentration that elicited half maximal inhibition. For both equations, $h$ denoted the Hill coefficient.

\section{Computational studies overview}

A number of computational techniques were used in this study, to assist the reader in understanding the logistics of these methods, we have provided a summary flowchart (supplemental information: Figure S6). 


\section{Homology modelling of MurM}

Due to the natural ability of streptococci to undergo homologous recombination, S. pneumoniae MurM genes are highly mosaic, and so, in line with the enzymology studies, the MurM sequence used for homology modelling was that of $S$. pneumoniae MurM ${ }_{159}$. $S$. pneumoniae MurM, S. aureus FemX (PDB ID: 6SNR) and FemA (PDB ID: 1LRZ), and Weissella viridescens FemX (PDB ID: 3GKR) were aligned (supplemental information: Figure $\mathrm{S} 1$ ) by multiple sequence alignment using Clustal Omega to determine sequence identity (Sievers et al., 2011).

The structure of $S$. aureus FemX was used as the template for homology modelling due to its high relatedness with MurM. S. aureus FemX and MurM $_{159}$ sequences were aligned, and using MODELLER (Eswar et al., 2006; Martí-Renom et al., 2000; sali and Blundell, 1993; Fiser et al., 2000) a test model was generated to verify the validity of the template and the alignment. This model was evaluated by computing its energy profile according to the DOPE-HR (high-resolution version of the Discrete Optimized Protein Energy) (Shen and sali, 2006), smoothed via window averaging with a size of 15 residues. The profiles of template and model were compared (supplemental information: Figure S7), and further refinement was conducted in the region between Lys230 and Pro299, as well as in all loop regions. This optimisation was conducted by performing a very slow MD annealing on the selected regions, whilst maintaining the remaining structure. The scoring of the resulting conformations was obtained via a function built specifically to evaluate the geometry of loops. For this step, 64 different base models were created and their secondary structure was refined independently 16 times. The resulting 1024 models were evaluated and ranked using DOPE-HR as well as the SOAP (Statistically Optimized Atomic Potentials) (Dong et al., 2013). The 10 best scoring models for each score were selected and evaluated based on the number of physical constraint violations present.

The best model of MurM $\mathrm{M}_{159}$ was aligned with the previously published MurM model (Fiser et al., 2003) or W. viridescens Femx homologues (Fonvielle et al., 2013; Biarrotte-Sorin et al., 2004) for visualisation and analysis in PyMOL (Version 2.1.0).

\section{Molecular docking of truncated Lipid II to MurM}

A truncated Lipid II substrate (supplemental information: Figure S2) was created for initial molecular docking simulations. The truncated Lipid II was drawn in ChemDraw Professional (Version 17.1) and converted to a pdb file using Avogadro (Version 1.2.0). To prepare the ligand file for docking, the protonation state in $\mathrm{H}_{2} \mathrm{O}$ at $\mathrm{pH} 7.4$ was computed. Subsequently the equilibrium geometry minimizing the potential energy was computed using the general amber force field (GAFF) (Wang et al., 2004) from within the Avogadro2 software (Hanwell et al., 2012). Molecular docking was conducted using AutoDock Vina (Trott and Olson, 2010), for which pdbqt files were generated from the pdb files of receptor model and ligands using AutoDock Tools (Morris et al., 2009). Initially the location of the binding site was verified by providing the algorithm with a search space that included the entire protein. Docking was then repeated by restricting the search space to the identified binding site, in order to obtain the final docked conformation.

Molecular docking of Lipid II, Cardiolipin, Phosphatidylglycerol and Phosphatidylethanolamine to the putative MurM binding site.

Using the HADDOCK2.4 web server (Van Zundert et al., 2016), full length Lipid II, cardiolipin and phosphatidylglycerol and phosphatidylethanolamine were docked into the putative MurM binding site, where the binding site is defined by the residues Lys35, Trp38, Phe103, Arg215 and Tyr219.

\section{Coarse-grained molecular dynamics simulations}

All coarse-grained simulations were carried out with the GROMACS package (Version 2018) and the Martini (Version 2.2) forcefield (Abraham et al., 2015; de Jong et al., 2012). Simulations at the coarse-grained and atomstic resolutions were carried out at $313 \mathrm{~K}$. For coarse-grained simulations, a stochastic velocity rescale thermostat with a coupling constant of 1:0 ps controlled the temperature.

The coordinates of the MurM homology model were used to generate a coarse-grained model using the 'martinise.py' script (de Jong et al., 2013). The protein was coarse grained to the EINeDyn model (Periole et al., 2009) with an elastic network strength and cutoff of $500 \mathrm{kJmol}^{-1} \mathrm{~nm}^{-2}$ and 0:9 nm, respectively. The Lipid II model for inclusion in the membrane was parameterised using a united atom model (Gromos 53a6) generated by the Auto-mated topology builder (ATB) web-interface (Malde et al., 2011). Following this, the coarse-grained mapping was pursued iteratively, and the bonded terms were fitted with PyCGTOOL (Graham et al., 2017).

Since the pneumococcal membrane comprises a complex mixture of lipids, a simplified membrane composition was required for the simulations. In order to elucidate the effects of phosphatidylglycerol and cardiolipin on MurM, a non-pneumococcal lipid, phosphatidylethanolamine, was used as the majority lipid. Simulations were conducted with three different membrane systems (supplemental information: Table S1). System 1 comprised phosphatidylethanolamine and phosphatidylglycerol in a molar ratio of $75 \%$ and $25 \%$ respectively, system 2 contained phosphatidylethanolamine, phosphatidylglycerol and cardiolipin in a molar ratio of $76 \%, 16 \%$ and $8 \%$ respectively and system 3 comprised phosphatidylethanolamine, phosphatidylglycerol and cardiolipin at a molar ratio of $72 \%, 12 \%$ and $16 \%$ respectively. The membrane systems of size $\sim 16 \times 16 \times 11: 5 \mathrm{~nm}$ were generated with the CHARMM-GUI web interface (Jo et al., 2017). Each system was relaxed with a series of minimisation and equilibration steps with timesteps of 5-20 fs, for up to $30 \mathrm{~ns}$. The equilibration steps utilised a semi-isotropic Berendsen barostat, with a 4:0 ps coupling constant (Berendsen et al., 1984). Following equilibration, Lipid II molecules (10 in total) were added to each membrane. The systems were then minimised and equilbriated (for $10 \mathrm{~ns}$ ), followed by a $2 \mu$ s production run to ensure sufficient mixing of all the lipid components. All production runs were carried out using a $10 \mathrm{fs}$ timestep and a Parrinello-Rahman semi-isotropic barostat with a 12 ps coupling constant (Parrinello and Rahman, 1981). The Lennard-Jones potential was cutoff using the Potential shift Verlet scheme at long 
ranges. The reaction field method (Tironi et al., 1995) was used for electrostatics calculations, with dielectric constants of 15 and infinity for charge screening in the short- and long-range regimes, respectively. The short-range cutoff for non-bonded and electrostatic interactions was $1: 2 \mathrm{~nm}$. Once lipid mixing was ensured, the size of each system was increased to $\sim 32 \mathrm{~nm}$ in the dimension perpendicular to the membrane and MurM was added in a random orientation around $8 \mathrm{~nm}$ above each membrane. Biologically relevant salt concentrations $(0.15 \mathrm{M} \mathrm{NaCl})$ were added and $10 \%$ of the water molecules were changed to antifreeze particles to prevent localised freezing during simulations. After minimisation and $1 \mathrm{~ns}$ of equilibration, during which the protein backbone was restrained with $1000 \mathrm{kJmol}^{-1} \mathrm{~nm}^{-2}$ harmonic restraints, $6 \times 5 \mu$ s production runs were generated per membrane composition (supplemental information: Table S1).

\section{All-atom molecular dynamics simulations}

Atomistic simulations were conducted using the CHARMM36m forcefield (Huang et al., 2017). The Lipid II model used here was also used in previous work (Witzke et al., 2016), while all other lipid models were obtained from the CHARMM-GUI membrane builder module (Jo et al., 2008). For each coarse-grained membrane system, two repeats were chosen where: 1) the last frame of the production run had a distinct orientation of MurM, relative to the membrane 2) MurM adhered to the membrane surface (supplemental information: Table S2). The last frame of the chosen coarse-grained repeats were then backmapped to the all-atom model, using the backward script (Wassenaar et al., 2014). Unfavourable ring conformers were corrected by carrying our minimisation and equilibration steps with dihedral restraints of $25000 \mathrm{kJmol}^{-1} \mathrm{rad}^{-2}$ on key ring torsions. After the transformation was carried out, each system was cropped in the $z$ dimension to a height of $16: 5 \mathrm{~nm}$, to remove unnecessary $\mathrm{H}_{2} \mathrm{O}$ molecules.

Each system was minimised and equilibrated for a total of $1 \mathrm{~ns}$, while the backbone of the protein was restrained with $1000 \mathrm{kJmol}^{-1} \mathrm{~nm}^{-2}$ harmonic restraints. Two production runs of $250 \mathrm{~ns}$ were carried out for each system. During the production runs a timestep of 2 fs was used, and the pressure (1 bar) regulated with a semi-isotropic Parrinello-Rahman barostat, with a coupling constant of 5:0 ps. The Lennard-Jones potential was cutoff with the Force-switch modifier from 1:0 to 1:2 nm. The short range cutoff for the electrostatic interaction was also 1:2 nm and the Particle mesh Ewald (PME) algorithm (Darden et al., 1993) was used for the long-range regime.

Analysis was carried out over the final $100 \mathrm{~ns}$ of each simulation, unless stated otherwise. All simulations were visualised using Visual Molecular Dynamics (VMD) or PyMOL (Version 2.2.0) (Humphrey et al., 1996). Other analysis tools were written with a combination of GROMACS tools and in house scripts, that utilised the python module MDAnalysis (Gowers et al., 2016). The depletion/enrichment (D-E) indices were determined by first counting the number of lipids with a centre of geometry within $1.1 \mathrm{~nm}$ of the protein and then comparing this number to the number expected in the bulk of the membrane, using the procedure described by Corradi et al. (2018). The D-E index was obtained by dividing the lipid composition in the $1.1 \mathrm{~nm}$ shell around the protein by the bulk membrane composition. Thus a $D-E$ index $>1$ indicates enrichment, while a $D-E$ index $<1$ indicates depletion. The $D-E$ index was determined for the last $100 \mathrm{~ns}$ of each simulation in $50 \mathrm{~ns}$ blocks for all repeats. For a given membrane composition, $8 \mathrm{D}$-E indices were obtained for each lipid, from which the average and standard errors were calculated. The enrichment maps were generated by first determining the 2D density map of the membrane using the GROMACS tool densmap. Following this, the enrichment percentage was determined using the procedure described by Corradi et al. (2018). An enrichment percentage $<0 \%$ indicated that local membrane composition was depleted with respect to the bulk membrane composition. The code for the 2D enrichment maps and D-E indices was reported in Shearer et al. (2019).

\section{QUATIFICATION AND STATISTICAL ANALYSIS}

Crystallographic statistics were calculated using software/programs as described in the methods, and values are reported in Table 1. Enzyme assays were performed in duplicate, generating data that differed by no more than $10 \%$. Average values were then plotted. The standard errors of the fits of constants defining the relationships between the response of MurM to phospholipid and phospholipid concentration according to equations 1 and 2 were calculated by GraphPad Prism (Version 8.4.1). 